\title{
Momentum correction techniques for neoclassical transport in stellarators
}

\author{
H. Maaßberg*, C.D. Beidler, and Y. Turkin \\ Max-Planck Institut für Plasmaphysik, \\ EURATOM Association, D-17491 Greifswald, Germany
}

(Dated: August 6, 2009)

\begin{abstract}
In the traditional neoclassical ordering for stellarators, mono-energetic transport coefficients are evaluated using the simplified Lorentz form of the pitch-angle collision operator which violates momentum conservation. In this paper, the parallel momentum balance with radial parallel momentum transport and viscosity terms is analysed, in particular with respect to the radial electric field. Next, the impact of momentum conservation in the stellarator $\operatorname{lmfp}$-regime is estimated for the radial transport and the parallel electric conductivity. Two different momentum correction techniques are described based on mono-energetic transport coefficients calulated by the DKES code [W.I. van Rij and S.P. Hirshman, Phys. Fluids B 1, 563 (1989)]. The benchmarking of the parallel electric conductivity and of the bootstrap current is presented for a tokamak as well as for two W7-X stellarator configurations [G. Grieger et al., Phys. Fluids B 4, 2081 (1992)]. Finally, the impact of the momentum correction on the expected total bootstrap current is briefly analysed for two W7-X scenarios.
\end{abstract}

\section{INTRODUCTION}

For stellarators, the computations of even mono-energetic neoclassical transport coefficients are rather time consuming in the lmfp-regime. Several numerical tools are available: DKES [1, 2], which is based on the Fourier-Legendre expansion of the linearised drift-kinetic equation, and quite different Monte Carlo techniques; see Refs. [3-7]. All these codes have been extensively benchmarked within the International Collaboration on Neoclassical Transport in Stellarators for all stellarator configurations of interest; see Ref. [8]. The treatment of the linearised collision operator with momentum conservation, however, would require the solution of the drift kinetic equation in 4D-phase space instead of the 3D mono-energetic solution. Consequently, momentum correction techniques based on the mono-energetic transport coefficients become very attractive. Both techniques described in this paper are based on databases of the three mono-energetic transport coefficients, determined with respect to radius, collisionality and radial electric field for each magnetic configuration precalculated by the DKES code.

The moment equation approach is a traditional technique for calculating the neoclassical transport in tokamaks $[9,10]$. From the drift kinetic equation, the parallel particle and heat viscosities are estimated depending on the plasma flows which are defined by the parallel momentum and heat balances. By the flux-friction relations, the radial particle and heat fluxes are related to the thermodynamic forces, i.e. the gradients. Since the parallel viscosities are a symmetric moment of the distribution function with respect to $v_{\|}$, a simplified collision term can be used for the estimation of these quantities without affecting the momentum conservation, which is important for the parallel flows. The viscosity coefficients have been estimated analytically for the different transport regimes in tokamaks. The moment equation approach has also been applied to stellarators [11]. For general stellarator configurations, however, the parallel flows have been estimated only in limiting cases, for example in the collisionless limit where the bootstrap current density is independent of the radial electric field.

Taguchi [12] extended the moment equation approach to a momentum correction technique for general stellarator configurations; for a nearly equivalent technique see Refs. $[13,14]$. Both methods use the mono-energetic transport coefficients calculated from the drift kinetic equation with a simple pitch-angle collision term without momentum conservation instead of viscosity coefficients for the different collisionality regimes. Furthermore, the radial electric field is included in both approaches with the approximation of an incompressible $\mathbf{E} \times \mathbf{B}$ flow. These techniques are extremely attractive since a very complex stellarator geometry, i.e. a broad $B_{m n}$ Fourier spectrum in Boozer coordinates, can be treated by the numerical tools used for the calculation of the mono-energetic transport coefficients, but the complexity of $B$ has no effect on the momentum correction itself. These moment equation methods allow also for the correction of the radial particle and heat fluxes. In Ref. [12], the Sonine (associated Laguerre) expansion of the distribution function and linearised collision operator included only the first two polynomials and the moments were defined with brevity of notation in mind. In the generalisation of Taguchi's approach described here in Sec. V, the moments are redefined which, as in the Sugama-Nishimura formalism [13, 14], leads to intrinsic ambipolarity of the particle fluxes for axisymmetric tokamaks at each order of expansion (as shown in Appendix B). In this respect, the generalisation of [12] described here is different than what used in [14] and it is therefore possible that its convergence properties will also be different (this is currently undergoing investigation).

An additional "direct" momentum correction technique as described in Sec. IV is based also on the mono-energetic transport coefficients, but can be applied only to the parallel flows. This technique is in one sense the generalisation of the classical

\footnotetext{
*E-mail: maass@ipp.mpg.de
} 
Spitzer problem [15] based on the first Legendre moment of the full linearised collision operator. Here, a system of integrodifferential equations with respect to the velocity is solved which is coupled for all particle species. In general, this approach can be also applied for electron cyclotron (ECCD) and neutral beam (NBCD) current drive calculations based on the adjoint approach [16, 17].

The paper is organised as follows. In Sec. II, the simplified linearised drift kinetic equation and the mono-energetic transport coefficients are defined. Then, the mono-energetic parallel momentum balances are analysed, in particular the parallel momentum transport coefficient dependent on the radial electric field is discussed. Special attention is given to large radial electric fields for which the banana orbits and, consequently, the parallel viscosity disappear in tokamaks. Results are shown both for a tokamak and a W7-X configuration. Furthermore, the impact of parallel flows on the radial transport is analysed. In particular for the stellarator $\operatorname{lmfp}$ transport, it is shown that this impact is negligible. Sec. III derives analytically the Spitzer problem in the collisionless asymptotic limit from the linearised drift kinetic equation following Refs. [16, 17]. In this limit it is shown that the parallel viscosity can be reformulated as the friction of passing and trapped particles.

Sec. IV describes the "direct" momentum correction technique, which is rather equivalent to a generalised Spitzer problem. In Sec. V, the extension of Taguchi's momentum correction technique is described and the equations for the parallel flow moments and the correction terms for the radial transport are given. In Sec. VI, the benchmarking of both momentum correction techniques for the parallel electric conductivity and the bootstrap current density is described both for a tokamak and two W7-X configurations with a low and a high toroidal mirror term. For the tokamak examples, additional models are included in the benchmarking. Since momentum correction can increase or decrease the bootstrap current depending on the density and temperature gradients, the total bootstrap currents with and without momentum correction are calculated for both W7-X configurations using a predictive transport code [18]; these results are described in Sec. VII. The W7-X stellarator, which has no ohmic transformer, relies on the compensation of the minimised bootstrap current (which is one of the optimisation criteria) by ECCD to guarantee control of the edge configuration and proper operation of its island divertor. This aspect leads to the request of fairly accurate predictions both of the bootstrap current and the ECCD. Finally, a summary and conclusions are given in Sec. VIII.

\section{MONO-ENERGETIC TREATMENT}

The starting point is the drift-kinetic equation (DKE) which is linearised with respect to the 1st-order distribution function defined as the (small) deviation from the 0th-order (unshifted) Maxwellian, $F_{M}$, with the density, $n(r)$, the electrostatic potential, $\Phi(r)$, and the temperature, $T(r)$, assumed to be constant on flux-surfaces. The 1st-order DKE becomes inhomogeneous with a radial driving force, $-\dot{r} F_{M}^{\prime}$ ( $r$ being the radius, $\dot{r}$ the radial component of the $\nabla B$-drift velocity and $F_{M}^{\prime}$ the radial derivative of the Maxwellian with total energy conserved), and with a parallel driving force, $\propto v_{\|} B F_{M}$; see e.g. Ref. [1]. Splitting this DKE with respect to the driving forces leads to two $1 s t$-order distribution functions, $f$ and $g$, where $f$ is related to $-\dot{r} F_{M}^{\prime}$ (symmetric in $v_{\|}$) and $g$ to $v_{\|} B F_{M}$. The Vlasov operator couples symmetric and asymmetric terms; consequently, $f(g)$ has also asymmetric (symmetric) contributions.

In order to solve the 1st-order DKE, the derivatives of $f$ and $g$ with respect to the radius, $r$, and the absolute velocity, $v$, are assumed to be negligible with respect to the corresponding derivatives of the Maxwellian. In addition, the collision operator is simplified by the Lorentz form of the pitch-angle collision term which violates like-particle momentum conservation. This approach allows for an independent solution for each particle species, $\alpha$. Here, the species index $\alpha$ is dropped only for the mono-energetic treatment presented in this Section. Finally, the component of the $\nabla B$-drift within the flux surface is omitted, and the incompressible approximation, $\mathbf{E} \times \mathbf{B} /\left\langle B^{2}\right\rangle$ where $\langle\ldots\rangle$ is the flux-surface average, is used for the $\mathbf{E} \times \mathbf{B}$-drift which is not reliable at large radial electric fields [19]. This approach offers the important advantage that $(\mathbf{B} \times \boldsymbol{\nabla} B) \cdot \mathbf{E}$ may be neglected in both acceleration terms, $\dot{p}$ and $\dot{v}\left(p=v_{\|} / v\right.$ is the pitch), allowing a conservative formulation in which $\dot{v} \equiv 0$. With these approximations, $r$ and $v$ are only parameters in this local and mono-energetic DKE. The 1 st-order distribution functions are now split in two portions

$$
f=f_{1}+f_{2} \quad \text { and } \quad g=g_{1}+g_{2}
$$

where $f_{1}$ and $g_{1}$ are the solutions of the simplified DKE without momentum conservation

$$
\mathcal{V}\left(f_{1}\right)-\nu \mathcal{L}\left(f_{1}\right)=-\dot{r}\left(A_{1}+x^{2} A_{2}\right) F_{M} \quad \text { and } \quad \mathcal{V}\left(g_{1}\right)-\nu \mathcal{L}\left(g_{1}\right)=p v b A_{3} F_{M},
$$

with the normalised velocity, $x=v / v_{t h}$, and with the normalised magnetic field strength, $b=B / B_{0}$, where for $B_{0}$ the $(m, n)=(0,0)$ Fourier mode of $B$ in Boozer co-ordinates is used. The radial component of the $\nabla B$-drift is given by

$$
\dot{r}=\frac{T}{q} \frac{1+p^{2}}{B^{3}} x^{2}(\mathbf{B} \times \nabla B) \cdot \nabla r
$$


In eq. (1), $f_{2}$ and $g_{2}$ represent the result of the momentum correction techniques (only the $1 s t$ Legendre term of the flux-surfaceaveraged corrections are used; see below). The thermodynamic forces $A_{i}$ for the particle species $\alpha$ are defined by

$$
A_{1}=\frac{n^{\prime}}{n}-\frac{q E_{r}}{T}-\frac{3}{2} \frac{T^{\prime}}{T}, \quad A_{2}=\frac{T^{\prime}}{T} \quad \text { and } \quad A_{3}=\frac{q}{T} \frac{\langle\mathbf{E} \cdot \mathbf{B}\rangle}{\left\langle B^{2}\right\rangle} B_{0} ;
$$

where the prime represents the raidal derivative (the notation being nearly equivalent to [2]). $\mathcal{V}$ is the (local and mono-energetic) Vlasov operator

$$
\mathcal{V}=\left(\frac{p v}{B} \mathbf{B}+\frac{\mathbf{E} \times \mathbf{B}}{\left\langle B^{2}\right\rangle}\right) \cdot \nabla_{\mathbf{s}}-v \frac{1-p^{2}}{2 B^{2}} \mathbf{B} \cdot \nabla B \frac{\partial}{\partial p}
$$

where $\mathbf{E}=-\nabla \Phi$ is the radial electric field, and $\nabla_{\mathbf{s}}$ the gradient within the flux surface. In eq. (2), $\mathcal{L}$ is the Lorentz form of the pitch-angle collision operator

$$
\mathcal{L}=\frac{1}{2} \frac{\partial}{\partial p}\left(1-p^{2}\right) \frac{\partial}{\partial p}
$$

and $\nu(v)$ the collision frequency. For the treatment of the mono-energetic transport coefficients, both the thermodynamic forces and the Maxwellian are dropped in the following by introducing the definitions $f_{1}=\hat{f}_{1}\left(A_{1}+x^{2} A_{2}\right) F_{M}$ and $g_{1}=\hat{g}_{1} A_{3} F_{M}$.

The DKES code [1, 2] is used to solve eq.(2). DKES (Drift Kinetic Equation Solver) is based on a Fourier-Legendre expansion of the 1st-order distribution functions, $\hat{f}_{1}$ and $\hat{g}_{1}$, with respect to the poloidal and toroidal angles (in magnetic co-ordinates) and the pitch, $p=v_{\|} / v$. For DKES, the magnetic configuration is described by the 2D-Fourier spectrum in Boozer co-ordinates for each flux surface. The stellarator free-boundary 3D-equilibria are calculated with the VMEC code [20] and the Fourier spectrum in Boozer co-ordinates with the JMC code [21]. The W7X examples of this paper are current-free vacuum configurations (see [8]). The (traditional) mono-energetic transport coefficients calulated by DKES are defined by the following moments with $[A]=\int_{-1}^{1}\langle A\rangle d p / 2: D_{11}=\left[\dot{r} \hat{f}_{1}\right]$ is the particle diffusion coefficient, $D_{31}=v\left[b p \hat{f}_{1}\right]$ the bootstrap current coefficient, $D_{13}=\left[\dot{r} \hat{g}_{1}\right]$ the Ware pinch coefficent, and $D_{33}=v\left[b p \hat{g}_{1}\right]$ the electric conductivity coefficient. Onsager symmetry leads to the relation $D_{31}=-D_{13}$.

\section{A. Parallel momentum balances}

The parallel mono-energetic momentum balances are obtained by multiplying the DKE (2) by $p b$, taking the flux-surface average and performing the pitch integration. For completeness, the radial component of the Vlasov operator is taken into account. With the notational abbreviation $\widehat{\mathcal{F}}_{1}=\left(\hat{f}_{1}, \hat{g}_{1}\right)$, this leads to

$$
\begin{aligned}
\frac{1}{V^{\prime}} \frac{d}{d r} V^{\prime}\left[b \dot{r} p \widehat{\mathcal{F}}_{1}\right] & -\frac{v}{B_{0}}\left[\mathbf{B} \cdot \boldsymbol{\nabla} \ln b P_{2}(p) \widehat{\mathcal{F}}_{1}\right] \\
& -\frac{1}{\left\langle B^{2}\right\rangle}\left[(\mathbf{E} \times \mathbf{B}) \cdot \nabla b p \widehat{\mathcal{F}}_{1}\right]+\nu\left[b p \widehat{\mathcal{F}}_{1}\right]={ }_{\frac{1}{3} v\left\langle b^{2}\right\rangle}^{0}
\end{aligned}
$$

where $V$ is the volume and $P_{2}(p)=\left(3 p^{2}-1\right) / 2$ is the Legendre polynomial of degree 2 . The 1 st term in this parallel momentum balance describes the radial transport of parallel momentum which, in general, is ignored in the traditional neoclassical theory (see e.g. the review [22]). In an axisymmetric configuration (with circular or elliptical cross section) with $E_{r}=0$, the $\sin \theta$ part of $\hat{f}_{1}$ (symmetric in $p$ ) leads to the particle transport whereas the $\cos \theta$ part (asymmetric in $p$ ) finally leads to the bootstrap current coefficient. This separation is broken at larger $E_{r}$ : the $\sin \theta$ component of $\hat{f}_{1}$ has also asymmetric contributions leading to radial transport of parallel momentum.

The parallel momentum transport coefficients in mono-energetic form, [ $b \dot{r} p \widehat{\mathcal{F}}_{1}$ ], are estimated with a new extended version of DKES. In Fig. 1, the mono-energetic $D_{p 1}=\left[b \dot{r} p \hat{f}_{1}\right]$ (normalised to the particle transport coefficient $D_{11}=\left[\dot{r} \hat{f}_{1}\right]$ ) and $D_{p 3}=\left[b \dot{r} p \hat{g}_{1}\right]$ (normalised to the Ware pinch coefficient $D_{13}=\left[\dot{r} \hat{g}_{1}\right]$ ) are shown for rather large $E_{r}$ normalised to the toroidal resonance value, $E_{r}^{*}=E_{r} / E_{r}^{\text {res }}$ with $E_{r}^{\text {res }}=t \epsilon_{t} v B_{0}$ where $t$ is the rotational transform and $\epsilon_{t}=r / R_{0}$ where $R_{0}$ is the major radius, for a tokamak configuration (on the left) and for a W7-X configuration at half the plasma radius (on the right). The value of the collisionality, $\nu^{*}=\nu R / t v$, places these results in the lmfp-regime.

For an axisymmetric configuration where both $D_{11}$ and $D_{13}$ are independent of $E_{r}$ (strictly for $E_{r}^{*} \ll 1$ ), $D_{p 1} / D_{11}$ slightly exceeds $D_{p 3} / D_{13}$, and both terms scale linearly in $E_{r}^{*}$. For $E_{r}^{*}>1$, all these radial transport coefficients $\left(D_{11}, D_{p 1}, D_{31}\right.$ and $\left.D_{p 3}\right)$ are strongly reduced. For stellarators, the situation is fairly equivalent. For the large $E_{r}^{*}$, the stellarator specific lmfp-regimes for radial transport, i.e. the $1 / \nu$-, the $\sqrt{\nu}$ - and the $\nu$-regime, are strongly suppressed. The radial transport in 

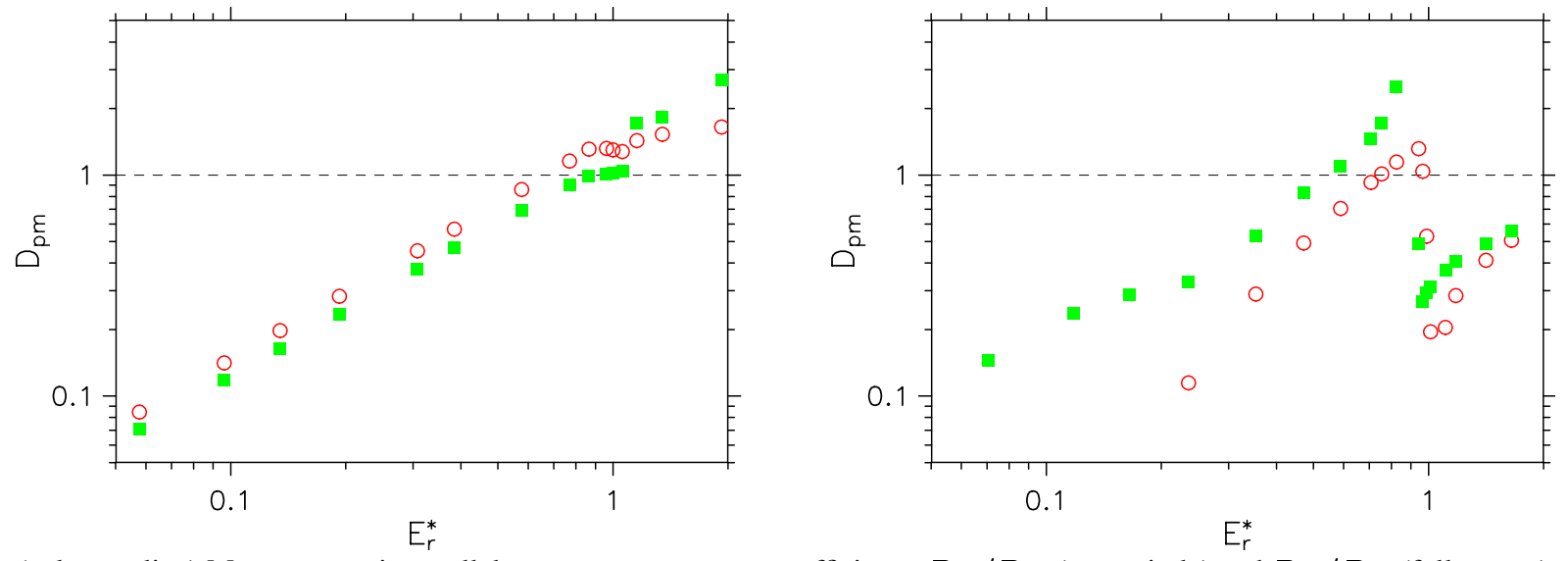

FIG. 1: (colour online) Mono-energetic parallel momentum transport coefficients, $D_{p 1} / D_{11}$ (open circle) and $D_{p 3} / D_{13}$ (full square), vs. the normalised radial electric field, $E_{r}^{*}$, for a tokamak with circular cross-section $\left(\epsilon_{t}=0.1\right.$ and $\nu^{*}=1.9 \cdot 10^{-4}$ on the left $)$ and for the "standard" W7-X configuration at half the plasma radius $\left(\epsilon_{t}=0.048\right.$ and $\nu^{*}=6.3 \cdot 10^{-4}$ on the right $)$.

these stellarator regimes is dominated by the large symmetric component of $\hat{f}_{1}$ for $E_{r}^{*} \ll 1$, which does not contribute to the parallel momentum transport. (For $E_{r}^{*}>1$, helical resonances equivalent to the toroidal one appear, and the radial transport coefficients disappear only for $E_{r}^{*} \gg 1$.) For the W7-X example of Fig. 1, the momentum transport coefficient $D_{p 1}$ changes sign for $E_{r}^{*}<0.2$ (inward transport).

Neglecting the parallel momentum transport ( $1 s t$ term) in eq. (6), the momentum balance within the flux surface is analysed. The $2 n d$ term is the mono-energetic form of the parallel viscosity, and the $3 r d$ term is the perpendicular viscosity driven by the $\mathbf{E} \times \mathbf{B}$ flow. The parallel viscosity is related to the pressure anisotropy (reflected by the $P_{2}(p)$ moment of the 1 st order distribution functions, $\hat{f}_{1}$ and $\hat{g}_{1}$ ). The perpendicular viscosity, however, is related to the parallel flow (the $\mathbf{E} \times \mathbf{B}$ term in the Vlasov operator is symmetric with respect to $p$ ). For both momentum balance equations (6), the flows driven by the radial $\left(\hat{f}_{1}\right)$ and parallel $\left(\hat{g}_{1}\right)$ thermodynamic forces, given by $v\left[b p \hat{f}_{1}\right]$ and $v\left[b p \hat{g}_{1}\right]$, must be balanced by both viscosities. The inhomogeneous part of $\hat{g}_{1}$ corresponding to the collisional limit where both viscosities vanish, is eliminated in the representation of Figs. 2 and 3 (on the right). The mono-energetic parallel conductivity $D_{33}=v\left[b p \hat{g}_{1}\right]$ is used to define the effective circulating (passing) particle fraction, $f_{c}^{\text {eff }}=1-f_{t}^{\text {eff }}=3 \nu D_{33} / 2 v v_{t h}$; see Section IV. In the collisionless limit, $\nu \rightarrow 0, f_{t}^{\text {eff }}=f_{t}$ where $f_{t}$ is the (standard) trapped particle fraction defined below in eq. (12) whereas $f_{t}^{\text {eff }}=0$ in the collisional limit, $\nu \rightarrow \infty$. Both limits are independent of the particle species.
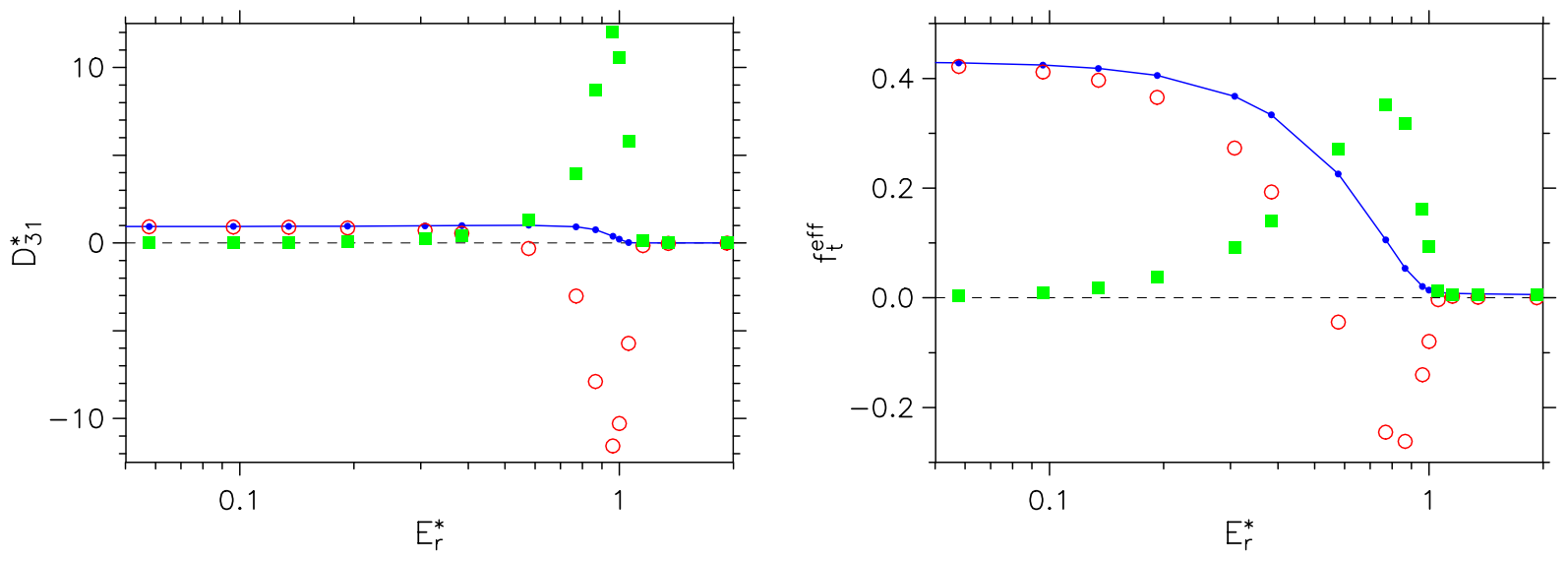

FIG. 2: (colour online) Parallel (open circle) and perpendicular (full square) viscosities as well as the parallel transport coefficients (dots connected by line) vs. the normalised radial electric field, $E_{r}^{*}$, for a tokamak with circular cross-section $\left(\epsilon_{t}=0.1\right.$ and $\left.\nu^{*}=1.9 \cdot 10^{-4}\right)$. On the left, for $\hat{f}_{1}$ and on the right, for $\hat{g}_{1}$.

As an example, Fig. 2 shows all contributions (except the radial momentum transport) to the mono-energetic momentum balances (6) for $\hat{f}_{1}$ (on the left) and for $\hat{g}_{1}$ (on the right) for a tokamak. $D_{31}$ as well as both viscosities are normalised to the collisionless limit of a large-aspect-ratio tokamak with circular cross section, $D_{31}=2 f_{t} v_{t h} / 3 \omega_{c} t \epsilon_{t}$ for $\epsilon_{t} \ll 1$ with $f_{t} \simeq 1.46 \sqrt{\epsilon_{t}}$ and $\omega_{c}=q B_{0} / m$. For $E_{r}^{*} \ll 1$, the perpendicular $\hat{f}_{1}$-viscosity is negligible and the parallel flow corresponding to the bootstrap current, which is related to the $D_{31}$ coefficient, is balanced only by the parallel $\hat{f}_{1}$-viscosity. At $E_{r}^{*}$ close to 1 , 
both $\hat{f}_{1}$-viscous terms become very large, but nearly compensate each other, and for $E_{r}^{*}>1$ all three terms in the momentum balance strongly decrease with $E_{r}$. A rather similar feature is found for the $\hat{g}_{1}$-momentum balance (on the right), but the $\hat{g}_{1^{-}}$viscous terms at $E_{r}^{*} \simeq 1$ are much less than in the case of $\hat{f}_{1}$ ( $f_{t}=0.458$ for the collisionless limit). For the large $E_{r}^{*}>1$, the trapped particles, i.e. the banana orbits, disappear and, consequently, the viscous damping effects are suppressed. Exactly this feature does not exist in stellarators (see below).
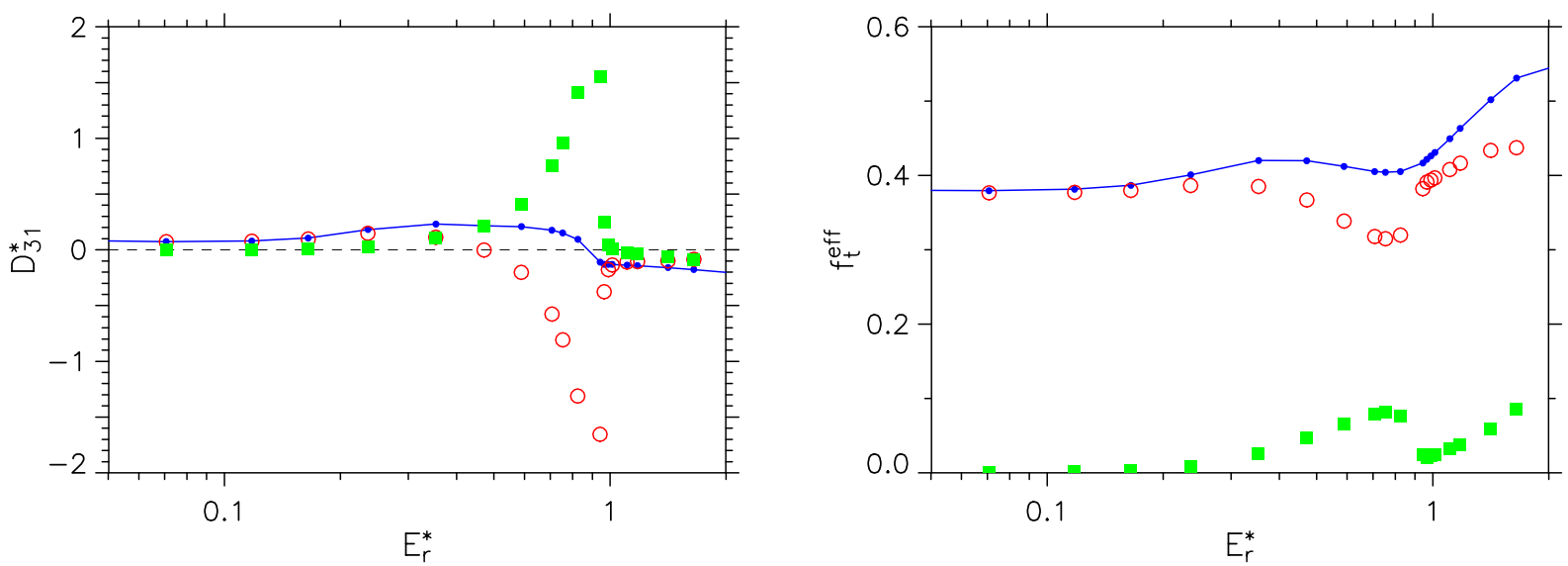

FIG. 3: (colour online) Parallel (open circle) and perpendicular (full square) viscosities as well as the parallel transport coefficients (dots conneted by line) vs. the normalised radial electric field, $E_{r}^{*}$, for the "standard" W7-X configuration at half the plasma radius $\left(\epsilon_{t}=0.048\right.$ and $\nu^{*}=6.3 \cdot 10^{-4}$ ). On the left, for $\hat{f}_{1}$ and on the right, for $\hat{g}_{1}$.

Again, the situation is rather similar for the W7-X configuration for $E_{r}^{*}<1$; see Fig. 3. The significantly reduced $\hat{f}_{1}$-viscosity terms as well as the normalised $D_{31}$ (on the left) reflect the W7-X optimisation criterion of minimised bootstrap current for small $E_{r}^{*}$ (more than an order of magnitude smaller then in the equivalent axisymmetric configuration for this example). For $E_{r}^{*}>1$, the toroidal resonance effects disappear and the helical effects dominate (the helical $(1,1)$ resonance is about $5 \cdot E_{r}^{\text {res }}$ ), with the $D_{31}$ coefficient becoming negative. For the $\hat{g}_{1}$-momentum balance, the perpendicular viscosity is significantly smaller than in the tokamak case in this $E_{r}^{*}$ range. As the main difference, however, the parallel viscosity as well as the effective trapped particle fraction, $f_{t}^{\text {eff }}$ (small circles and solid line), even increase for $E_{r}^{*}>1$ ( $f_{t}=0.432$ for the collisionless limit). In 3D stellarator configurations, trapped particles effects cannot be suppressed and dominate the parallel viscous damping of the flows within the flux surface (see also Sec. III).

These results lead to a strong restriction for the radial electric field: only for $E_{r}^{*} \ll 1$, does the radial transport of parallel momentum remain negligible. This restriction is stronger than the one related to the validity of the incompressible $\mathbf{E} \times \mathbf{B}$ approximation; see [19]. Furthermore, the perpendicular viscosity (related to the $\mathbf{E} \times \mathbf{B}$ flow) can be ignored for $E_{r}^{*} \ll 1$. For these conditions, there is no principal difference between tokamaks and stellarators (in a co-ordinate system at rest): trapped particle effects dominate the viscous damping of the parallel flows.

\section{B. Radial transport}

Here, the impact of momentum correction on the radial transport is analysed. For a tokamak, the radial transport and the friction are connected due to the relation (which is absent in non-symmetric stellarator configurations [23])

$$
\mathbf{B} \cdot \boldsymbol{\nabla} B=-t \epsilon_{t}(\mathbf{B} \times \boldsymbol{\nabla} B) \cdot \boldsymbol{\nabla} r .
$$

Using this tokamak relation and multiplying the DKE (2) by $p / b$ leads to a slightly different form of the momentum balance of eqs. (6), the radial momentum transport term as well as the perpendicular viscosity term are omitted here.

$$
\left[\frac{p}{b} \mathcal{V}\left(\widehat{\mathcal{F}}_{1}\right)-\frac{p}{b} \mathcal{L}\left(\widehat{\mathcal{F}}_{1}\right)\right]=-\omega_{c} \frac{t \epsilon_{t}}{v}\left[\dot{r} \widehat{\mathcal{F}}_{1}\right]+\nu\left[\frac{p}{b} \widehat{\mathcal{F}}_{1}\right]=\frac{0}{3} v
$$

Neglecting finite aspect ratio effects $\left(\epsilon_{t} \ll 1\right)$, a relation between the parallel viscosity and the radial flux is obtained by comparison with eqs. (6),

$$
\frac{v^{2}}{B_{0}}\left[\mathbf{B} \cdot \nabla \ln b P_{2}(p) \widehat{\mathcal{F}}_{1}\right] \simeq \omega_{c} t \epsilon_{t}\left[\dot{r} \widehat{\mathcal{F}}_{1}\right]
$$


Taking also the Onsager symmetry into consideration, all transport coefficients, i.e. the radial transport, the bootstrap current and the parallel conductivity coefficients, are linked together via the flux-friction relations; see e.g. [10]. These relations hold also for a momentum conserving collision operator; see e.g. [24]. Consequently, the impact of momentum conservation on the parallel flows (bootstrap and conductivity) affects also the radial fluxes in tokamaks.

In the stellarator $\operatorname{lmfp}$-regimes, the situation is completly different. Here, the ripple-trapped particles dominate the radial transport, and their distribution function is nearly symmetric allowing for a bounce-averaged formulation of the DKE for calculating the radial transport; see e.g. [25, 26]. In the lmfp-regime, the parallel viscosity evaluated for $\hat{f}_{1}$ is proportional to $\nu^{*}$ (see eqs.(6)) and only weakly dependent on $E_{r}$. Although this term is also determined by the symmetric component of $\hat{f}_{1}$, the large $D_{11}=\left[\dot{r} \hat{f}_{1}\right]$ has a quite different dependence on $\nu^{*}$ and $E_{r}$, e.g. in the $1 / \nu$ - and the $\sqrt{\nu}$-regimes. If any distribution function is split into a slow and a fast time scale with respect to bounce-averaging, the slow part determines $D_{11}$ [25], but does not contribute to the parallel viscosity. This can be shown [11] by formulating the flux-surface averaging as bounce-averaging for the ripple-trapped particles, and the slow contribution vanishes due to the $\mathbf{B} \cdot \boldsymbol{\nabla} B$ term (this is different from $\mathbf{B} \times \nabla B$ in $\dot{r}$ ). Consequently, the impact of momentum conservation on the radial transport in the stellarator lmfp-regime is negligible. At high collisionalities, however, the impact of the ripple-trapped particles on the radial transport becomes negligible, and momentum correction techniques can also affect the radial transport as in tokamaks.

Following Ref. [11], the poloidal component of the momentum balance couples the radial particle transport to the poloidal viscosity, $\left\langle\mathbf{B}_{\boldsymbol{p}} \cdot \boldsymbol{\nabla} \cdot \boldsymbol{\pi}\right\rangle$ (or equivalently for the toroidal components), where $\boldsymbol{\pi}$ is the stress tensor. In this fluid approach, the radial current of each particle species requires a perpendicular (Lorentz) force within the flux-surface which is attributed to the poloidal (toroidal) viscosity for the stellarator lmfp-regime. With the ambipolarity condition on the radial particle fluxes which requires that the radial current must vanish, also this non-parallel viscosity contribution in the total poloidal (toroidal) momentum balance vanishes. As a consequence, the stellarator $\operatorname{lmfp}$-transport regimes (e.g. the $1 / \nu$ - or the $\sqrt{\nu}$-regime) do not affect the flows within the flux-surface.

\section{MOMENTUM BALANCE IN THE COLLISIONLESS ASYMPTOTIC LIMIT}

The $2 n d$ term in the parallel momentum balances eqs. (6) is the parallel viscosity describing the damping of parallel flows due to the magnetic field inhomogenity. Here, the $2 n d$ Legendre coefficient of the distribution function appears in an equation for the 1 st Legendre coefficient for describing the parallel flows. This general formulation has the drawback that this form of the momentum balances cannot be directly extended for collision operators with momentum conservation. In the asymptotic collisionless limit, however, the 1 st-order DKE for $g_{1}$ (with the parallel driving force) is directly integrable for sufficiently small $E_{r}$. This approach is important for the reformulation of the parallel viscosity as the friction of passing with trapped particles and, consequently, will be briefly described here following Refs. [16, 17]. The particle species index $\alpha$ must be introduced at this point since the interaction with all other species $\beta$ becomes important.

The local pitch, $p$, is transformed to the (normalised) global magnetic moment, $\lambda$, by

$$
p=\sqrt{1-\lambda b_{\max }} \quad \text { with } \quad b_{\max }=B / B_{\max }
$$

where $B_{\max }$ is the maximum of $B$ on the flux surface. Note, that $\lambda$ is only strictly an invariant of motion corresponding to the local and mono-energetic Vlasov operator of eq. (4) (3D-phase space) for $E_{r}=0$. Consequently, the collisionless approach described here holds only for $E_{r}^{*} \ll 1$; compare also Fig. 3 (on the right). Taguchi's momentum conserving collision operator $[16,17]$ is defined by

$$
C^{\alpha}\left(g^{\alpha}\right)=\nu^{\alpha} \mathcal{L}_{\lambda}\left(g^{\alpha}\right)+p C_{l=1}^{\alpha}\left(g_{l=1}^{\alpha}\right)+\nu^{\alpha} p g_{l=1}^{\alpha}
$$

where $g_{l=1}^{\alpha}$ is the 1 st Legendre term of the 1 st order distribution function $g^{\alpha}, C_{l=1}^{\alpha}$ the 1 st Legendre term of the full linearised collision operator (interaction with all other species $\beta$ included), and $\mathcal{L}_{\lambda}$ is the Lorentz form of the pitch-angle collision operator of eq. (5) transformed to $\lambda$

$$
\mathcal{L}_{\lambda}=\frac{2}{b_{\max }} \sqrt{1-\lambda b_{\max }} \frac{\partial}{\partial \lambda} \lambda \sqrt{1-\lambda b_{\max }} \frac{\partial}{\partial \lambda} .
$$

The last term in eq. (8) is the 1 st Legendre term of $-\mathcal{L}$ with respect to $p$ and compensates $\mathcal{L}_{\lambda}$ for momentum conservation. In $\lambda$, the simplified DKE with modified r.h.s. corresponding to eq. (2), now with momentum conservation, takes the form $\left(p=\sqrt{1-\lambda b_{\max }}\right)$

$$
v p \frac{\partial g^{\alpha}}{\partial s}-C^{\alpha}\left(g^{\alpha}\right)=\nu_{0}^{\alpha} x p b_{\max } F_{M}
$$


where $s$ is the co-ordinate along the field line, $\nu_{0}^{\alpha}$ is the thermal collision frequency, and $x=x^{\alpha}=v / v_{t h}^{\alpha}$ is the normalised velocity of species $\alpha$. In the limit $\nu^{\alpha} \rightarrow 0$, the integrability constaint $\partial g^{\alpha} / \partial s \rightarrow 0$ and, consequently, $g^{\alpha}=\left\langle g^{\alpha}\right\rangle$ holds for the asymmetric component of $g^{\alpha}$. Furthermore, this component vanishes for trapped particles, i.e. for $\lambda \geq 1$.

$$
K_{g}^{\alpha}(x)=S_{g}^{\alpha}(x) \cdot e^{-x^{2}}=\frac{3}{2} \int_{0}^{1} g^{\alpha} d \lambda=\frac{3}{2 b_{\max }} \int_{-1}^{1} p g^{\alpha} d p
$$

where $S_{g}^{\alpha}(x)$ is the definition of the collisionless Spitzer function. With flux-surface averaging, eq. (9) reduces to a 1D equation for the velocity dependence of the asymmetric part of $g^{\alpha}$ in the collisionless limit

$$
g^{\alpha}(\lambda \leq 1)=\frac{3}{4} \frac{\left\langle b_{\max }^{2}\right\rangle}{f_{c}} K_{g}^{\alpha}(x) \int_{\lambda}^{1} \frac{d \lambda}{\left\langle\sqrt{1-\lambda b_{\max }}\right\rangle} \quad \text { and } \quad g^{\alpha}(\lambda \geq 1)=0
$$

and

$$
C_{l=1}^{\alpha}\left(K_{g}^{\alpha}\right)-\frac{f_{t}}{f_{c}} \nu^{\alpha}(x) K_{g}^{\alpha}=-\nu_{0}^{\alpha} x F_{M}
$$

and with the trapped particle fraction

$$
f_{t}=1-f_{c}=1-\frac{3}{4}\left\langle b_{\max }^{2}\right\rangle \int_{0}^{1} \frac{\lambda d \lambda}{\left\langle\sqrt{1-\lambda b_{\max }}\right\rangle}
$$

(where $f_{c}$ is the circulating particle fraction). Eq. (11) can be easily reformulated for the Spitzer function $S_{g}^{\alpha}(x)$ and the collisional limit, i.e. the classical Spitzer formulation [15] of the parallel conductivity, is obtained for $f_{t}=0$ and $\alpha=e$ (electrons). In an equivalent approach [27] for $f_{t} \neq 0$, the collisionless Spitzer function is obtained from a variational principle introduced in Ref. [28].

In this collisionless picture, only passing particles contribute to the parallel flow which is damped by the friction of the passing with the trapped particles. Whereas the formulation of the viscous damping term in eqs. (6) is based on the symmetric part of the distribution functions (i.e. the pressure anisotropy), eqs. (10) and (11) are only based on the $1 s t$ Legendre term, i.e. the coupling of the different Legendre harmonics by the Vlasov operator has disappeared. Consequently, this approach is well suited for momentum correction at very low collisionalities.

\section{IV. “DIRECT” MOMENTUM CORRECTION TECHNIQUE}

With the 1st-order distribution functions $\mathcal{F}_{1}^{\alpha}=\left(f_{1}^{\alpha}, g_{1}^{\alpha}\right)$, which are the solutions of the simplified DKE (2), the momentum corrected solutions of eq. (1) are defined by the DKE with the linearised momentum-conserving collision operator, $C^{\alpha}$, defined in eq. (8) but with $\mathcal{L}$ of eq. (5) instead of $\mathcal{L}_{\lambda}$ for each particle species $\alpha$

$$
\begin{aligned}
& \mathcal{V}\left(f^{\alpha}\right)-C^{\alpha}\left(f^{\alpha}\right)=-\dot{r}\left(A_{1}^{\alpha}+x^{2} A_{2}^{\alpha}\right) F_{M}^{\alpha} \\
& \mathcal{V}\left(g^{\alpha}\right)-C^{\alpha}\left(g^{\alpha}\right)=p v b A_{3}^{\alpha} F_{M}^{\alpha}
\end{aligned}
$$

where the thermodynamic forces $A_{i}^{\alpha}$ are given in eq. (3), and $\mathcal{V}$ is the local and mono-energetic Vlasov operator of eq. (4). Using eqs. (1) and (2), the corrections for momentum conservation, $\mathcal{F}_{2}^{\alpha}=\left(f_{2}^{\alpha}, g_{2}^{\alpha}\right)$, are given by

$$
\mathcal{V}\left(\mathcal{F}_{2}^{\alpha}\right)-C^{\alpha}\left(\mathcal{F}_{2}^{\alpha}\right)=C^{\alpha}\left(\mathcal{F}_{1}^{\alpha}\right)-\nu^{\alpha} \mathcal{L}\left(\mathcal{F}_{1}^{\alpha}\right)
$$

where $\mathcal{L}$ is given by eq. (5). Then, the momentum correction $\mathcal{F}_{2}^{\alpha}$ is directly related to the "error" in the momentum conservation with respect to $\mathcal{F}_{1}^{\alpha}$. Multiplying this equation with $p b$, performing the pitch integration and the flux-surface averaging leads to

$$
\left\langle b \int_{-1}^{1} p \mathcal{V}\left(\mathcal{F}_{2}^{\alpha}\right) d p\right\rangle-C_{l=1}^{\alpha}\left(\left\langle b \int_{-1}^{1} p \mathcal{F}_{2}^{\alpha} d p\right\rangle\right)=C_{l=1}^{\alpha}\left(\begin{array}{c}
\Gamma_{31}^{\alpha} \\
\Gamma_{33}^{\alpha}
\end{array}\right)+\nu^{\alpha} \begin{array}{r}
\Gamma_{31}^{\alpha} \\
\Gamma_{33}^{\alpha}
\end{array}
$$

with the (normalised) mono-energetic fluxes $\Gamma_{31}^{\alpha}=\left(A_{1}^{\alpha}+x^{2} A_{2}^{\alpha}\right) D_{31}^{\alpha} F_{M}^{\alpha} / v_{t h}^{\alpha}$ and $\Gamma_{33}^{\alpha}=A_{3}^{\alpha} D_{33}^{\alpha} F_{M}^{\alpha} / v_{t h}^{\alpha}$. Neglecting both the radial momentum transport and the perpendicular viscosity term (related to $(\mathbf{E} \times \mathbf{B}) \cdot \nabla B$ ), the averaged Vlasov term can be 
expressed by the parallel viscosity related to $\mathcal{F}_{2}^{\alpha}$ equivalent to eq. (6). In this formulation, however, the Vlasov operator couples to the $2 n d$ Legendre coefficients of $\mathcal{F}_{2}^{\alpha}$ whereas the linearised collision operator is only based on the $1 s t$ ones.

Comparing eq. (14) with the equivalent collisionless problem of eq. (11), the function $\mathcal{K}_{2}^{\alpha}(x)$ is defined by

$$
\mathcal{K}_{2}^{\alpha}(x)=\left\langle\frac{b}{2} \int_{-1}^{1} p \mathcal{F}_{2}^{\alpha} d p\right\rangle,
$$

and equivalently $\mathcal{K}_{1}^{\alpha}(x)$ by the mono-energetic fluxes, $\mathcal{K}_{1}^{\alpha}=\left(\Gamma_{31}^{\alpha}, \Gamma_{33}^{\alpha}\right)$. Furthermore, the parallel viscosity is strictly identified in the limit $\nu \rightarrow 0$, but can be generalised by introducing the effective trapped particle fraction, $f_{t}^{\text {eff }}$,

$$
\left\langle\frac{b}{2} \int_{-1}^{1} p \mathcal{V}\left(\mathcal{F}_{2}^{\alpha}\right) d p\right\rangle=\frac{f_{t}^{\text {eff }}(x)}{f_{c}^{\text {eff }}(x)} \nu^{\alpha}(x) \mathcal{K}_{2}^{\alpha}(x),
$$

and $f_{t}^{\text {eff }}(x)$ is defined using the mono-energetic conductivity coefficient, $D_{33}$, by

$$
f_{t}^{\mathrm{eff}}=1-f_{c}^{\mathrm{eff}}=1-\frac{\nu^{\alpha}}{v v_{t h}^{\alpha}} D_{33}^{\alpha}\left(\frac{\nu^{\alpha} R}{v t}\right)
$$

with the mono-energetic "collisionality", $\nu^{\alpha} R / v t$ ( $R$ is the major radius). This definition guarantees the limits $f_{t}^{\text {eff }}(x \rightarrow 0)=0$ and $f_{t}^{\text {eff }}(x \rightarrow \infty)=f_{t}$ given in eq. (12). With eqs. (15) and (16), the momentum correction $\mathcal{K}_{2}^{\alpha}(x)$ is defined by the 1D integro-differential equation

$$
C_{l=1}^{\alpha}\left(\mathcal{K}_{2}^{\alpha}\right)-\frac{f_{t}^{\text {eff }}}{f_{c}^{\text {eff }}} \nu^{\alpha} \mathcal{K}_{2}^{\alpha}=-C_{l=1}^{\alpha}\left(\mathcal{K}_{1}^{\alpha}\right)-\nu^{\alpha} \mathcal{K}_{1}^{\alpha}
$$

With $\mathcal{K}^{\alpha}=\mathcal{K}_{1}^{\alpha}+\mathcal{K}_{2}^{\alpha}$, the final form (equivalent to a generalised Spitzer problem) for both the bootstrap current and the parallel conductivity component of $\mathcal{K}^{\alpha}$ is obtained

$$
C_{l=1}^{\alpha}\left(\mathcal{K}^{\alpha}\right)-\frac{f_{t}^{\text {eff }}}{f_{c}^{\text {eff }}} \nu^{\alpha} \mathcal{K}^{\alpha}=-\frac{1}{f_{c}^{\text {eff }}} \nu^{\alpha} \mathcal{K}_{1}^{\alpha}
$$

for each particle species $\alpha$. For the parallel conductivity, the r.h.s. reduces by using eq. (17) to $-x A_{3}^{\alpha} F_{M}^{\alpha}$ being equivalent to the collisionless limit of eq. (11). The formulas for $C_{l=1}^{\alpha}$ are given in Appendix A.

Next, the concept of the effective trapped particle fraction is analysed. For both the collisionless and the collisional limit, eqs. (17) and (18) give the correct momentum correction for the parallel flows with the magnetic field inhomogeneity taken into account. In the mono-energetic treatment, $1-f_{t}^{\text {eff }}$ reflects the contribution of the trapped particles to the parallel flow: the asymmetry of the trapped-particle distribution function with respect to $p$ depending on the ratio of the collision to the bounce frequency must be calculated from the solution of the corresponding DKE. This can be done analytically for both limits, and is obtained e.g. from DKES for the general case. For the momentum correction, the local details of the distribution function are not relevant, the important global effect is given by $f_{t}^{\text {eff }}$ related to the parallel conductivity coefficient $D_{33}^{\alpha}$ corresponding to eq. (17).

Fig. 4 shows $f_{t}^{\text {eff }}$ versus the (mono-energetic) collisionality for the W7-X "standard" configuration and for the equivalent axisymmetric configuration with only the poloidal Fourier modes, $B_{m, n=0}$, taken into account. First, the ripple-trapped particles in W7-X significantly contribute to $f_{t}^{\text {eff }}$. The fairly strong elongation of W7-X leads to a rather small $f_{t}^{\text {eff }}$ for the equivalent tokamak. Second, the ratio of the bounce to the collision frequency is very different for both configurations: the bounce frequency of the trapped particles in the stellarator ripple is much higher than in the corresponding tokamak case. Consequently, trapped-particle effects extend to much higher collisionalities in stellarators. Finally, the confidence intervals are given in Fig. 4 showing the Imfp-convergence problem of DKES for stellarator configurations: e.g. 1457 Fourier modes and 250 Legendre polynomials have been used at the lowest collisionality. These problems do not appear at the equivalent collisionalities for tokamak configurations.

Solving the coupled system (for electrons and ions) of eq. (18), the electron-ion cross-coupling terms in the linearised collision operator, $C_{l=1}^{\alpha}$, must be taken into account since both parallel flows are linked. Then, the bootstrap, $j_{b}^{\alpha}$, and the inductive $\left(E_{\|}\right.$ driven), $j_{i n d}^{\alpha}$, current densities are defined by

$$
j_{i n d}^{\alpha}=\frac{4}{\sqrt{\pi}} q^{\alpha} n^{\alpha} v_{t h}^{\alpha} \int_{0}^{\infty} \mathcal{K}^{\alpha}(x) x^{3} d x .
$$


with $x=x^{\alpha}=v / v_{t h}^{\alpha}$ and the parallel flow moments

$$
n^{\alpha} U_{k}^{\alpha} \equiv \int d^{3} v v_{\|} L_{k}^{(3 / 2)}\left(x^{2}\right) \bar{f}^{\alpha} .
$$

For the first two Sonine polynomials $L_{0}^{(3 / 2)}\left(x^{2}\right)=1$ and $L_{1}^{(3 / 2)}\left(x^{2}\right)=5 / 2-x^{2}$, the lowest order parallel flow moments are related to the parallel flow velocity, $V_{\|}^{\alpha}=U_{0}^{\alpha}$, and the parallel heat flux density, $q_{\|}^{\alpha}=-n^{\alpha} T^{\alpha} U_{1}^{\alpha}$. The $\ell=1$ Legendre moment of the linearised collision term couples the friction coefficients with the parallel flow moments

$$
\begin{gathered}
\mathcal{C}_{1}^{\alpha \beta}\left(\varphi_{1}\right)=\frac{3 m^{\alpha} v}{T^{\alpha}} F_{M}^{\alpha} \sum_{i} \frac{(2 i) ! !}{(2 i+3) ! !} a_{i} L_{i}^{(3 / 2)}\left(x^{2}\right) \\
\text { with } \quad a_{i}=\frac{3}{\tau^{\alpha \beta}} \sum_{j} \frac{(2 j) ! !}{(2 j+3) ! !}\left(U_{j}^{\alpha} M_{i j}^{\alpha \beta}+U_{j}^{\beta} N_{i j}^{\alpha \beta}\right)
\end{gathered}
$$

with the Braginski collision time, $\tau^{\alpha \beta}=3 \sqrt{\pi} / 4 \nu^{\alpha \beta}$. The matrix elements of the friction coefficients, $M_{i j}^{\alpha \beta}$ and $N_{i j}^{\alpha \beta}$, are defined by

$$
\begin{aligned}
& \frac{n^{\alpha}}{\tau^{\alpha \beta}} M_{i j}^{\alpha \beta}=\int d^{3} v v_{\|} L_{i}^{(3 / 2)}\left(x^{2}\right) \mathcal{C}^{\alpha \beta}\left(\frac{m^{\alpha} v_{\|}}{T^{\alpha}} L_{j}^{(3 / 2)}\left(x^{2}\right) f_{M}^{\alpha}, f_{M}^{\beta}\right) \\
& \frac{n^{\alpha}}{\tau^{\alpha \beta}} N_{i j}^{\alpha \beta}=\int d^{3} v v_{\|} L_{i}^{(3 / 2)}\left(x^{2}\right) \mathcal{C}^{\alpha \beta}\left(f_{M}^{\alpha}, \frac{m^{\beta} v_{\|}}{T^{\beta}} L_{j}^{(3 / 2)}\left(\left(x^{\beta}\right)^{2}\right) f_{M}^{\beta}\right)
\end{aligned}
$$

with $x^{\beta}=x v_{t h}^{\alpha} / v_{t h}^{\beta}$. The $M_{i j}^{\alpha \beta}$ describe the interaction of $\bar{f}^{\alpha}$ with the background Maxwellians (diffusive contribution) and the $N_{i j}^{\alpha \beta}$ the interaction of the Maxwellian of species $\alpha$ with the deviations from the Maxwellians, $\bar{f}^{\beta}$, of the background species (integral contribution).

With the incompressible approximation of the $\mathbf{E} \times \mathbf{B}$ drift in the Vlasov operator of eq. (4), the incompressibility condition of the parallel flow moments (only for the $g^{\alpha}$ portion of $\bar{f}^{\alpha}$ allowing for the Pfirsch-Schlüter flows)

$$
\nabla \cdot \frac{\mathbf{B}}{B} \int d^{3} v v_{\|} g^{\alpha} A(x) \approx 0
$$

for an arbitrary function $A(x)$ is obtained which is a magnetic differential equation with the solution

$$
\int d^{3} v v_{\|} g^{\alpha} A(x)=\frac{B}{\left\langle B^{2}\right\rangle}\left\langle B \int d^{3} v v_{\|} g^{\alpha} A(x)\right\rangle .
$$

Following the approach of Refs. [12, 14], the $g_{1}$ eq. (2) is multiplied by $\bar{f}^{\alpha} L_{j}^{(3 / 2)}$ where $\bar{f}^{\alpha}=f^{\alpha}+g^{\alpha}$ of eq. (1) is the solution of the combined eq. (13), and integrated over the velocity space. Then, the adjoint properties of the Vlasov and the collision operators are used. The Sonine expansion coefficients of a function $A(x)$ are defined by

$$
\llbracket A(x) \rrbracket_{j}=\frac{4}{\sqrt{\pi}} \int_{0}^{\infty} d x x^{2} e^{-x^{2}} L_{j}^{(3 / 2)}\left(x^{2}\right) A(x),
$$

and tedious manipulation finally yields a system of linear equations for the flux-surface averaged parallel flow moments, $\left\langle b U_{k}^{\alpha}\right\rangle$ with $b=B / B_{0}$, for an arbitrary order of the Sonine polynomial expansion, $j_{x}$. For an arbitrary value of $j=0,1,2, \ldots, j_{x}$ one obtains the equation for each species $\alpha$

$$
\begin{aligned}
\sum_{k=0}^{k_{x}}\left[\langle b U _ { k } ^ { \alpha } \rangle \left\{\delta_{k j}-c_{k} \frac{1}{\left\langle b^{2}\right\rangle} \frac{m^{\alpha}}{T^{\alpha}}\right.\right. & \left(\llbracket \nu^{\alpha} L_{k}^{(3 / 2)} D_{33}^{\alpha}\right]_{j}+ \\
& \left.\left.\sum_{\ell=0}^{\ell_{x}} c_{\ell} \llbracket L_{\ell}^{(3 / 2)} D_{33}^{\alpha} \rrbracket_{j}\left\{\frac{M_{\ell k}^{\alpha \alpha}+N_{\ell k}^{\alpha \alpha}}{\tau^{\alpha \alpha}}+\sum_{\beta \neq \alpha} \frac{M_{\ell k}^{\alpha \beta}}{\tau^{\alpha \beta}}\right\}\right)\right\} \\
& \left.-c_{k} \frac{1}{\left\langle b^{2}\right\rangle} \frac{m^{\alpha}}{T^{\alpha}} \sum_{\beta \neq \alpha}\left\langle b U_{k}^{\beta}\right\rangle \sum_{\ell=0}^{\ell_{x}} c_{\ell} \llbracket L_{\ell}^{(3 / 2)} D_{33}^{\alpha} \rrbracket_{j} \frac{N_{\ell k}^{\alpha \beta}}{\tau^{\alpha \beta}}\right] \\
& =-\llbracket D_{31}^{\alpha} \rrbracket_{j} A_{1}^{\alpha}-\llbracket x^{2} D_{31}^{\alpha} \rrbracket_{j} A_{2}^{\alpha}-\llbracket D_{33}^{\alpha} \rrbracket_{j} A_{3}^{\alpha}
\end{aligned}
$$


where $\delta_{k j}$ is the Kronecker delta, $c_{j}=3(2 j) ! ! /(2 j+3) ! !$ and $k_{x}=\ell_{x}=j_{x}$ is the maximum order of the Sonine expansion $\left(j_{x}=2\right.$ for the results shown in Secs. VI and VII), and $\nu^{\alpha}=\sum_{\beta} \nu^{\alpha \beta}$. The order of this system of linear equations, i.e. $\left(j_{x}+1\right) \times$ the number of particle species, is fairly low and can be easily solved in the energy convolution algorithm of the mono-energetic fluxes. In eq. (23), the r.h.s. represents the Sonine coefficients of the parallel flows without momentum correction, and the 1.h.s. the impact of the (collisionality dependent) effective trapped particle fraction (implicitly given by the $D_{33}^{\alpha}$ Sonine coefficients); compare with eq. (18). The parallel current density of species $\alpha$ is given by

$$
j^{\alpha}=q^{\alpha} n^{\alpha}\left\langle b U_{0}^{\alpha}\right\rangle .
$$

In particular, $A_{1}^{\alpha}=A_{2}^{\alpha}=0$ in eq. (23) leads to the inductive and $A_{3}^{\alpha}=0$ to the bootstrap current densities equivalent to eq. (19).

The radial transport (defined by the mono-energetic $D_{11}^{\alpha}$ coefficient) is completely decoupled from the parallel flow moments described by eq. (23). On the other hand, the parallel flow moments affect the radial particle and energy transport as can be shown when the $f_{1}$ eq. (2) is multiplied by $\bar{f}^{\alpha}$ and $K \bar{f}^{\alpha}$ and integrated over velocity space. Again, the adjoint properties of the Vlasov and the collision operators are used. Straightforward calculation analogous to Refs. $[12,14]$ leads to the (flux-surface averaged) radial particle and energy flux densities, $\Gamma^{\alpha}$ and $Q^{\alpha}$, respectively,

$$
\begin{aligned}
& \frac{1}{n^{\alpha}}\left[\begin{array}{l}
\Gamma^{\alpha} \\
Q^{\alpha} / T^{\alpha}
\end{array}\right]=-\left[\begin{array}{l}
L_{11}^{\alpha} \\
L_{21}^{\alpha}
\end{array}\right] A_{1}^{\alpha}-\left[\begin{array}{l}
L_{12}^{\alpha} \\
L_{22}^{\alpha}
\end{array}\right] A_{2}^{\alpha}-\left[\begin{array}{l}
L_{13}^{\alpha} \\
L_{23}^{\alpha}
\end{array}\right] A_{3}^{\alpha} \\
& -\frac{1}{\left\langle b^{2}\right\rangle} \frac{m^{\alpha}}{T^{\alpha}} \sum_{k=0}^{k_{x}}\left\{c _ { k } \langle b U _ { k } ^ { \alpha } \rangle \left(\left[\begin{array}{c}
\llbracket \nu^{\alpha} D_{31}^{\alpha} \rrbracket_{k} \\
{\left[x^{2} \nu^{\alpha} D_{31}^{\alpha}\right]_{k}}
\end{array}\right]\right.\right. \\
& \left.\left.+\sum_{\ell=0}^{\ell_{x}} c_{\ell}\left[\llbracket \llbracket x^{2} D_{31}^{\alpha}\right]_{\ell}^{\alpha} \rrbracket_{\ell}\right]\left\{\frac{M_{\ell k}^{\alpha \alpha}+N_{\ell k}^{\alpha \alpha}}{\tau^{\alpha \alpha}}+\sum_{\beta \neq \alpha} \frac{M_{\ell k}^{\alpha \beta}}{\tau^{\alpha \beta}}\right\}\right) \\
& +\sum_{\beta \neq \alpha} c_{k}\left\langle b U_{k}^{\beta}\right\rangle \sum_{\ell=0}^{\ell_{x}} c_{\ell}\left[\llbracket\left[D_{31}^{\alpha} \rrbracket_{\ell}\right] \frac{N_{\ell k}^{\alpha \beta}}{\tau^{\alpha \beta}}\right\}+\frac{m^{\alpha}}{q^{\alpha}} \frac{\left\langle\widetilde{G}^{2}\right\rangle}{B_{0}^{2}} X_{\mathrm{PS}}^{\alpha}
\end{aligned}
$$

$$
\text { where } \begin{aligned}
L_{i j}^{\alpha} & =\frac{4}{\sqrt{\pi}} \int_{0}^{\infty} d x x^{2} D_{i j}^{\alpha} h_{i} h_{j} e^{-x^{2}} \quad \text { with } h_{1,3}=1 \text { and } h_{2}=x^{2} \\
\frac{\widetilde{G}}{B_{0}} & =B \int^{\ell} \frac{d \ell^{\prime}}{B} \nabla \cdot \frac{\mathbf{B} \times \nabla r}{B^{2}}-\frac{B}{\left\langle B^{2}\right\rangle}\left\langle B^{2} \int^{\ell} \frac{d \ell^{\prime}}{B} \nabla \cdot \frac{\mathbf{B} \times \nabla r}{B^{2}}\right\rangle .
\end{aligned}
$$

The $L_{i j}^{\alpha}$ are the thermal transport coefficients with direct energy convolution (see [1,2]), i.e. without momentum correction included. $\left\langle\widetilde{G}^{2}\right\rangle$ is the Pfirsch-Schlüter factor which is evaluated from the mono-energetic radial transport coefficients at very large collisionalities, $\left\langle\widetilde{G}^{2}\right\rangle=3 x\left(\omega_{c}^{\alpha}\right)^{2} D_{11}^{\alpha} / 2 \nu^{\alpha}\left(v_{t h}^{\alpha}\right)^{2}$ (independent of the particle species $\alpha$ ); compare eq. (55) of Ref. [13] at very high collisionalities. $X_{\mathrm{PS}}^{\alpha}$ represents an effective friction force related to the pressure and temperature gradients and is given by

$$
\begin{gathered}
X_{\mathrm{PS}}^{\alpha}=\sum_{\beta \neq \alpha}\left(\frac{\left(n^{\alpha} T^{\alpha}\right)^{\prime}}{n^{\alpha} q^{\alpha}}-\frac{\left(n^{\beta} T^{\beta}\right)^{\prime}}{n^{\beta} q^{\beta}}\right)\left(\left[\begin{array}{c}
1 \\
5 / 2
\end{array}\right] \frac{M_{00}^{\alpha \beta}}{\tau^{\alpha \beta}}-\left[\begin{array}{l}
0 \\
1
\end{array}\right] \frac{M_{10}^{\alpha \beta}}{\tau^{\alpha \beta}}\right) \\
-\frac{\left(T^{\alpha}\right)^{\prime}}{q^{\alpha}}\left(\left[\begin{array}{c}
1 \\
5 / 2
\end{array}\right] \sum_{\beta \neq \alpha} \frac{M_{01}^{\alpha \beta}}{\tau^{\alpha \beta}}-\left[\begin{array}{l}
0 \\
1
\end{array}\right]\left\{\frac{M_{11}^{\alpha \alpha}+N_{11}^{\alpha \alpha}}{\tau^{\alpha \alpha}}+\sum_{\beta \neq \alpha} \frac{M_{11}^{\alpha \beta}}{\tau^{\alpha \beta}}\right\}\right) \\
\quad-\sum_{\beta \neq \alpha} \frac{\left(T^{\beta}\right)^{\prime}}{q^{\beta}}\left(\left[\begin{array}{c}
1 \\
5 / 2
\end{array}\right] \frac{N_{01}^{\alpha \beta}}{\tau^{\alpha \beta}}-\left[\begin{array}{l}
0 \\
1
\end{array}\right] \frac{N_{11}^{\alpha \beta}}{\tau^{\alpha \beta}}\right)+\frac{2 T^{\alpha}}{3 q^{\alpha}}\left(\left[\begin{array}{c}
I_{0}^{\alpha} \\
I_{1}^{\alpha}
\end{array}\right] A_{1}^{\alpha}+\left[\begin{array}{c}
I_{1}^{\alpha} \\
I_{2}^{\alpha}
\end{array}\right] A_{2}^{\alpha}\right) \\
\text { with } \quad I_{n}^{\alpha} \equiv \frac{4}{\sqrt{\pi}} \int_{0}^{\infty} d x x^{2 n+4} e^{-x^{2}} \nu^{\alpha}(x) .
\end{gathered}
$$

All the correction terms in eq. (25) scale roughly linearly with the collision frequency and are small in the lmfp-regime. The $L_{i j}^{\alpha}$ contributions with $i, j=1,2$ reflect the stellarator $\operatorname{lm} f p$-transport, e.g. the $1 / \nu$ - and the $\sqrt{\nu}$ regimes. Consequently, momentum corrections are negligible at low collisionalities for stellarators; see also Sec. II B. At higher collisionalities, i.e. in the plateau and the Pfirsch-Schlüter regime, however, momentum correction affects the radial transport both in stellarators and tokamaks. The special case of the intrinsic ambipolarity of the radial particle fluxes in axisymmetric tokamaks is given in the Appendix B. 


\section{BENCHMARKING OF THE MOMENTUM CORRECTION TECHNIQUES}

Momentum correction techniques for stellarators must also be validated for tokamaks where several approaches exist. Fig. 5 shows the benchmarking of the parallel electric conductivity for a tokamak configuration (with circular cross section) at a moderate aspect ratio, $\epsilon_{t}=r / R=0.05$. Results of two tokamak approaches are shown: the Hinton-Hazeltine model [22], which assumes a very small $\epsilon_{t}$, and a fit representation to numerical Fokker-Planck calculations, abbreviated as SAL model [29] (with the $f_{t}$ representation of [30]). In addition, the collisionless limit based on the solution of eq. (11) is given. For the momentum correction based on moment equations (Sec. V), the small ion contribution is added to the parallel conductivity. At larger $\epsilon_{t}$, the Hinton-Hazeltine model deviates significantly, in particular at low collisionalities, whereas the agreement of the other approaches is good.

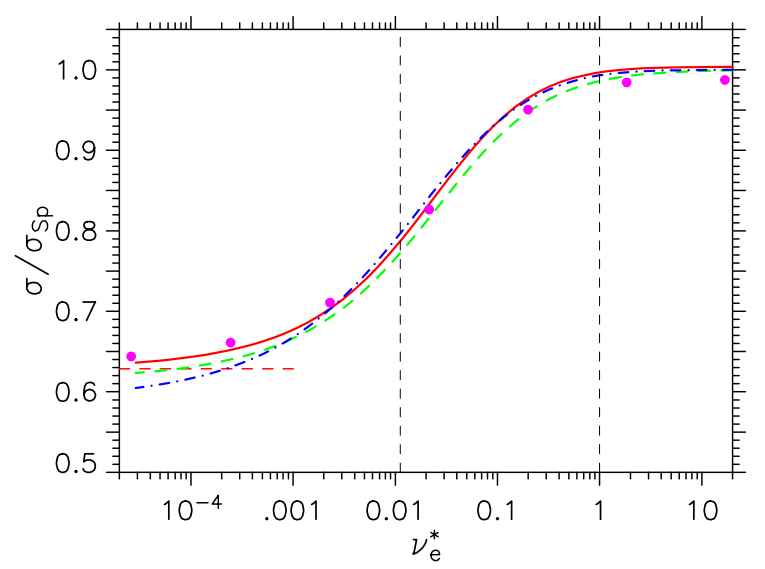

FIG. 5: (colour online) Parallel electric conductivity normalised to the Spitzer value vs. the (thermal) collisionality, $\nu_{e}^{*}=\nu^{e} R_{0} / v_{t h}^{e} t$ (different from the traditional tokamak definition), for a circular tokamak configuration with $\epsilon_{t}=0.05$. Momentum correction of Sec. IV (solid line) and of Sec. V (dots), the Hinton-Hazeltine model (dot-dashed line) and the SAL model (dashed line). The collisionless limit is given for reference. The transitions from the banana to the plateau and to the Pfirsch-Schüter regimes are indicated by the vertical dashed lines.

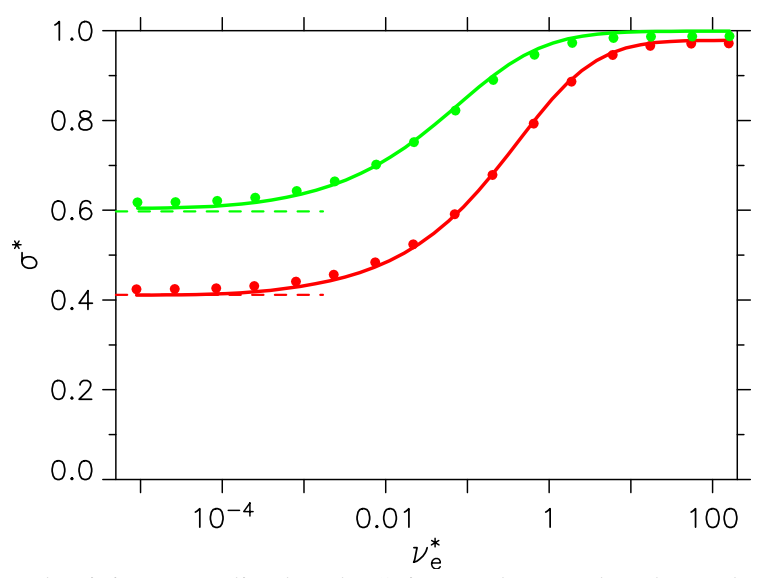

FIG. 6: (colour online) Parallel electric conductivity normalised to the Spitzer value vs. the (thermal) collisionality for the W7-X "low-mirror" (upper curve) and the "high-mirror" (lower curve) configurations both at half the plasma radius. Momentum correction of Sec. IV (solid lines) and of Sec. V (dots), the collisionless limits are indicated for reference.

The equivalent parallel electric conductivities are shown in Fig. 6 for the W7-X "low-" and "high-mirror" configurations at half the plasma radius $\left(\epsilon_{t} \simeq 0.05\right)$; the agreement of both momentum correction techniques is also good. The W7-X configurations are characterised by rather high elongation: 6.8 for the "low-" and 5.1 for the "high-mirror" case. Consequently, the helical as well as the toroidal ripple mainly determine the trapped particle fraction $\left(f_{t}=0.35\right.$ and 0.55 for the "low-" and the "high-mirror" case, respectively) as is also shown in Fig. 4 for the W7-X "standard" configuration. Note, that the reduction in the neoclassical conductivity starts at higher collisionalities compared to the tokamak case in Fig. 5. Consequently, "simple" tokamak formulas for the parallel electric conductivity are not sufficient for stellarator configurations even if the correct $f_{t}$ is used.

Next, the bootstrap current densities are benchmarked for the tokamak case with $\epsilon_{t}=0.05$. The bootstrap current densities 


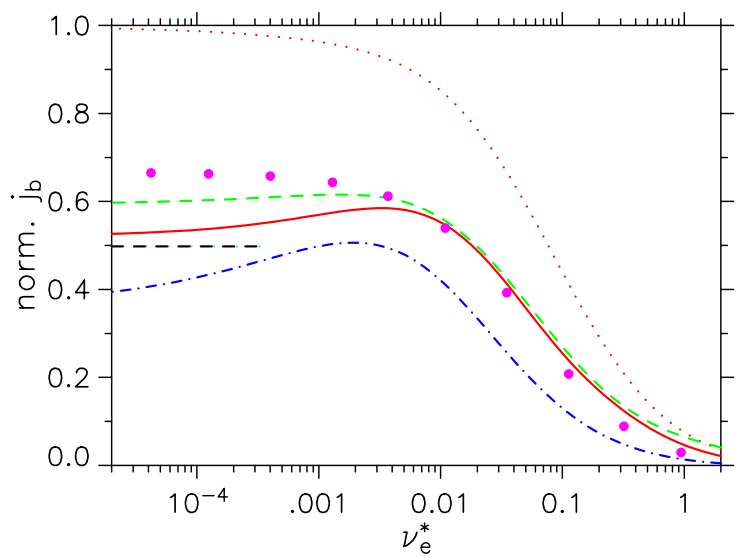

FIG. 7: (colour online) Bootstrap current density normalised to the collisionless limit without momentum correction vs. the (thermal) collisionality for a circular tokamak configuration with $\epsilon_{t}=0.05$. Momentum correction of Sec. IV (solid line) and of Sec. V (dots), the Hinton-Hazeltine model (dot-dashed line), the SAL model (dashed line) and the collisionless Hirshman model (on the left). The bootstrap current density without momentum correction (dotted line) is given for reference.

are normalised to the collisionless limit without momentum correction for a circular tokamak defined by

$$
j_{b}^{\mathrm{ref}}=-\sum_{\alpha} n^{\alpha} T^{\alpha} \frac{f_{t}}{\epsilon_{t} t B}\left(\frac{\left(n^{\alpha} T^{\alpha}\right)^{\prime}}{n^{\alpha} T^{\alpha}}-\frac{q^{\alpha} E_{r}}{T^{\alpha}}\right)
$$

with $f_{t} \simeq 1.46 \sqrt{\epsilon_{t}}$ for very small $\epsilon_{t}$. Fig. 7 shows the benchmarking for the tokamak case (equivalent to Fig. 5). In addition to the Hinton-Hazeltine model [22] and the SAL model [29], the collisionless Hirshman approach [31] is shown. Both momentum correction techniques of Sec. IV and V are in very reasonable agreement with these tokamak approaches. For this collisionality scan, $T^{e}=T^{i}, Z_{\mathrm{eff}}=1, n^{\prime} / n=-0.2 \mathrm{~m}^{-1}$ and $T^{e^{\prime}} / T^{e}=T^{i^{\prime}} / T^{i}=-1 \mathrm{~m}^{-1}$ are used. In the momentum correction techniques based on the moment equations, all contributions related to the radial electric field drop out for the tokamak case (intrinsic ambipolarity) whereas $E_{r}=0$ is used in the direct momentum correction. Here, $E_{r}$ affects only the individual $j_{b}^{\alpha}$, but the sum is independent of $E_{r}$. In the example of Fig. 7, the total bootstrap current density is significantly reduced due to the momentum conservation in the collision operator; compare with the case without momentum correction (dotted line). This reduction is only obtained if the temperature gradients dominate whereas even an increase of the bootstrap current density is found with momentum correction in the opposite case for dominating density gradients. For all these scenarios, very reasonable agreement with the tokamak models is found. This holds also for larger $\epsilon_{t}$ values (with exception of the Hinton-Hazeltine model).
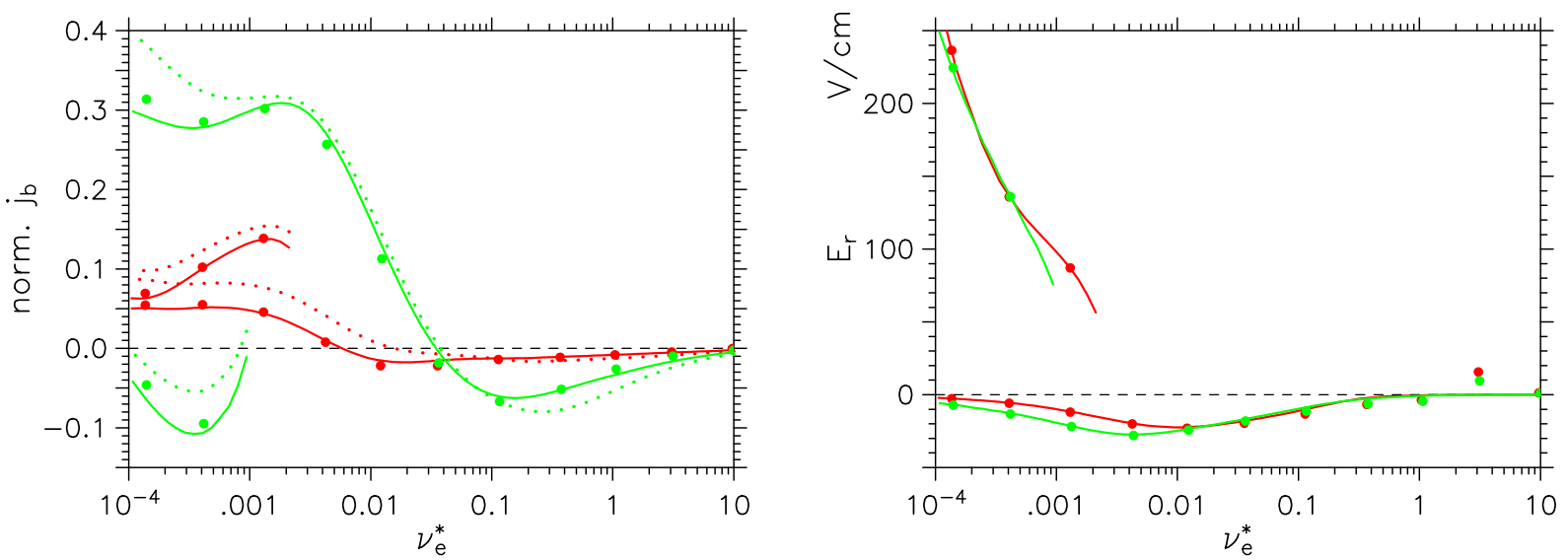

FIG. 8: (colour online) On the left: Bootstrap current density normalised to the collisionless limit of an equivalent circular tokamak without momentum correction vs. the (thermal) collisionality for the W7-X "low-mirror" (grey/green) and the "high-mirror" (black/red) configurations both at half the plasma radius. Momentum correction of Sec. IV (solid lines) and of Sec. V (dots); the bootstrap current densities without momentum correction (dotted lines) are given for referenece. On the right: the ambipolar radial electric field with momentum correction included (dots) and without (solid lines).

Finally, the benchmarking of both momentum correction techniques is briefly described for the W7-X configurations of Fig. 6. Fig. 8 shows the normalised bootstrap current density (on the left) and the corresponding radial electric field from the 
ambipolarity condition of the radial fluxes (on the right) both for the "low-" and the "high-mirror" configuration. As in the tokamak case, $T^{e}=T^{i}, Z_{\mathrm{eff}}=1, n^{\prime} / n=-0.2 \mathrm{~m}^{-1}$ and $T^{e^{\prime}} / T^{e}=T^{i^{\prime}} / T^{i}=-1 \mathrm{~m}^{-1}$ are used. Except for the high collisionalities, momentum correction has nearly no effect on the radial particle fluxes and, consequently, on the radial electric field (see also Sec. II B). At the low collisionalities, an additional "electron-root" is obtained (the unstable root in between is not shown) which, by evaluating the thermodynamic principle described in Refs. [32, 33], however, is not realised. For these W7-X configurations, the bootstrap current density is positive (tokamak-like) at low collisionalities and becomes negative at higher ones (the bootstrap current densities are always negative in a helically symmetric configuration). With respect to a reference tokamak scenario without momentum correction (as used for the normalisation), $j_{b}$ is reduced by roughy a factor of 3 for the "low-" and more than 10 for the "high-mirror" configuration. The effect of the momentum correction is of the same order as in the tokamak case, but with respect to the reduced bootstrap current densities of the W7-X configurations (compare with the scenario without momentum correction; dotted lines). Equivalent to the tokamak case, a dominant density gradient increases the momentum corrected $j_{b}$. Again, the agreement of both momentum correction techniques is very good.

In addition, the neoclassical ordering of Sec. II must be checked for the scenario shown in Fig. 8. First, the ion parallel flow (i.e. the ion bootstrap current density) is very small compared to the ion thermal velocity. Consequently, the assumption of a Maxwellian at rest is confirmed for the Oth-order distribution function. Second, the normalised radial electric field, here $E_{r}^{*}=$ $E_{r} / t \epsilon_{t} v_{t h}^{i} B_{0}$, is sufficiently small (largest $E_{r}^{*}=0.14$ for the electron-root at the lowest collisionality) and radial momentum transport can be neglected; see Sec. II A.

\section{BOOTSTRAP CURRENT SIMULATIONS FOR W7-X}

Momentum conservation in the collision operator leads to increased or decreased bootstrap current densities for dominating density or temperature gradients, respectively. The effect of momentum correction for the total bootstrap current predicted for the "low-" and the "high-mirror" W7-X configuration (compare Sec. VI) is described in this Section.

In the predictive transport simulations [18], the ion and electron energy balances with neoclassical heat fluxes are solved self-consistently with the ambipolar radial electric field. (Only at the outermost radii where the neoclassical fluxes are strongly decreased due to the low temperature, simple anomalous contributions are added). This assumption of mainly neoclassical transport leads to an upper limit for the temperatures and, consequently, for the bootstrap currents. The neoclassical particle and heat fluxes are evaluated from precalculated databases of mono-energetic transport coefficients (from DKES [1, 2]) by energy convolution with and without momentum correction included. The correction technique described in Sec. V requires only the solution of a small system of linear equations and is well suited for predictive transport simulations. Since the treatment of the particle balance must rely on anomalous transport modelling (the main recycling sources are at the outer radii with low neoclassical transport), fixed density profiles are assumed in these simulations. Modules for NBI and ECR heating and current drive are self-consistently coupled to the transport code [34, 35].
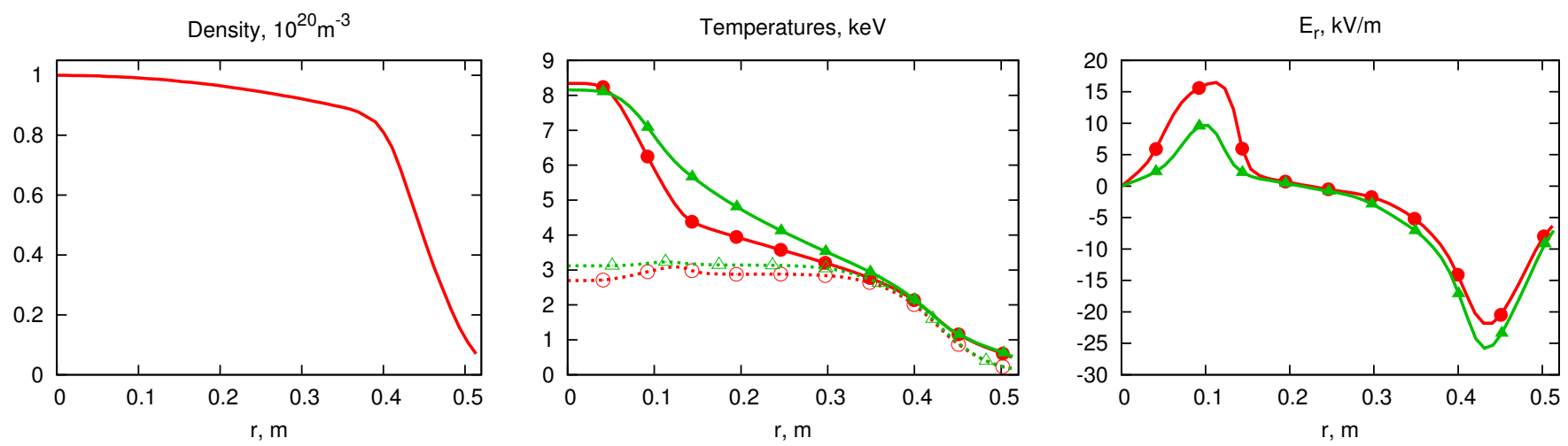

FIG. 9: (colour online) Profiles of density (left), temperatures (center, $T_{e}$ solid and $T_{i}$ dashed lines, respectively) and ambipolar radial electric field (right) for the W7-X "low-" (grey/green) and "high-mirror" (black/red) configurations.

As an example, Fig. 9 shows the density, the temperature and the ambipolar radial electric field profiles for both the "low-" and the "high-mirror" W7-X configurations. These simulations are performed with $10 \mathrm{MW}$ ECRH in the extra-ordinary mode (X2mode) with high absorption [36]. For both scenarios, the highly localised central power deposition results in an "electron-root" feature $[33,37]$ with positive radial electric fields, with steep electron temperature gradients and with flat ion temperature profiles (rather weak collisional coupling even at $10^{20} \mathrm{~m}^{-3}$ density). Due to the large ripple, the "electron-root" is more pronounced in the "high-mirror" configuration whereas the electron neoclassical confinement is degraded at larger radii, $r \geq 0.15 \mathrm{~m}$.

Also for this example, the ion parallel flow is very small with respect to the ion thermal velocity. The $E_{r}$ normalised to the 
ion resonance value, $E_{r}^{*}=E_{r} / t \epsilon_{t} v_{t h}^{i} B_{0}$, is fairly large: $E_{r}^{*}=0.48$ at $r=0.12 \mathrm{~m}$ for the "high-mirror" and $E_{r}^{*}=-0.27$ at $r=0.42 \mathrm{~m}$ for the "low-mirror" case in Fig. 9. Consequently, the situation becomes marginal, ion momentum transport (affecting the ion bootstrap current) driven by the thermodynamic forces $A_{1}^{i}$ and $A_{2}^{i}$ in eqs. (13) and (23) might play a role. At $r=0.12 \mathrm{~m}$, however, the electron contribution dominates the total bootstrap current density (the equivalent electron $E_{r}^{*}$ is very small). For the "low-mirror" scenario at $r=0.42 \mathrm{~m}$, the ion and the electron contributions are comparable. Taking ion momentum transport into account will reduce the ion bootstrap current density, but as a consequence of the collisional coupling, the electron bootstrap current density will be increased leading to a partial compensation with respect to the total bootstrap current density.
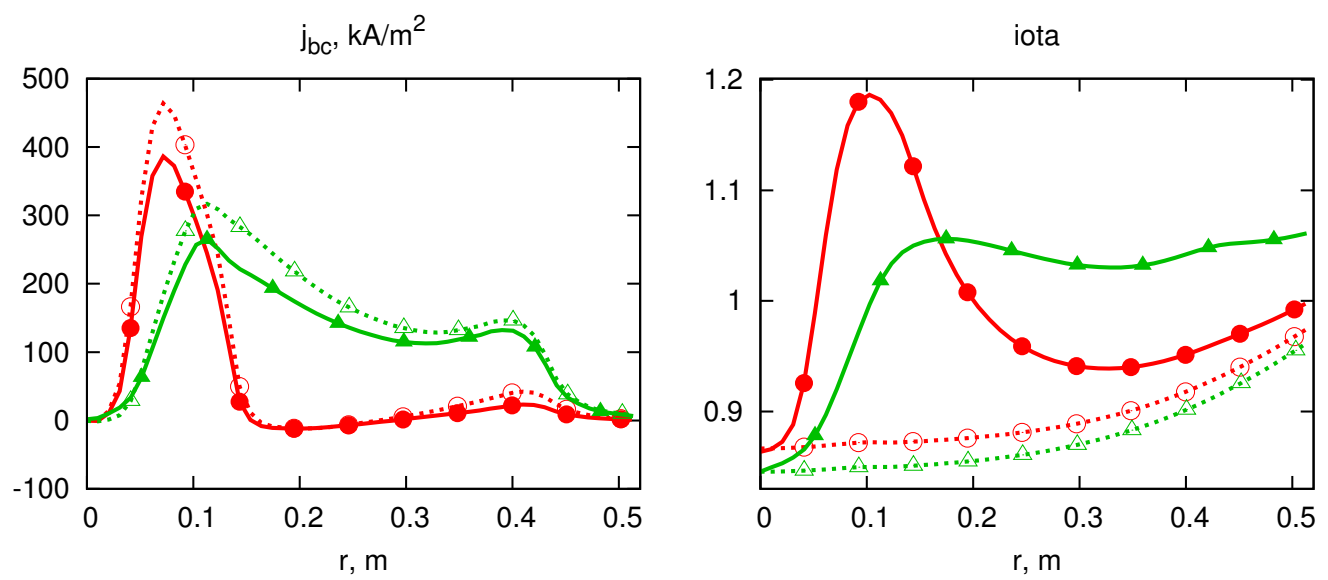

FIG. 10: (colour online) Left: Bootstrap current densities with momentum correction (solid lines) and without (dashed) for the W7-X "low-" (grey/green) and "high-mirror" (black/red) configurations. Right: Rotational transform profiles with momentum corrected bootstrap current (dashed lines: $t(r)$ without bootstrap current).

The effect of the momentum correction on the bootstrap current densities is shown in Fig. 10 (on the left). The bootstrap current density profile is fairly broad in the "low-mirror" configuration (total bootstrap current $I_{b}=88 \mathrm{kA}$ with momentum correction and $I_{b}=104 \mathrm{kA}$ without $)$ and highly localised within the "electron-root" region for the "high-mirror" case $\left(I_{b}=19.5\right.$ kA with momentum correction and $I_{b}=28.4 \mathrm{kA}$ without). This behaviour of the bootstrap current is in agreement with Fig. 8. The bootstrap current coefficients are strongly reduced for the "high-mirror" configuration for small radial electric fields, however this minimisation (one of the W7-X optimisation criteria) is partly lost for the "electron-root" scenario leading to the fairly strong peak in the bootstrap current density profile.

The impact of these bootstrap current density profiles on the rotational transform, $t$, are shown in Fig. 10 (on the right). The current profile contribution to the vacuum- $t(r)$ is evaluated with the susceptance coefficients [38] of the vacuum configuration. This approximation is reasonable as long as the impact of pressure and current on the shape of the flux surfaces is small. Furthermore, the bootstrap current densities are evaluated for the $t(r)$ of the vacuum configuration. In the $\operatorname{lm} f p$-regime, the bootstrap current coefficients scale roughly with $1 / t$ (i.e. tokamak-like) for the fixed Fourier spectrum of $B$. In both W7X scenarios of Fig. 10, the bootstrap current is too large, i.e. the edge $t$ is in conflict with a proper operation of the island divertor $[39,40]$. In principle, one might reduce the vacuum $t$ by the amount the bootstrap current supplies for stationary conditions, i.e. after several $L / R$-times. In particular for the "low-mirror" example, however, the shear $t$ ' is much too low leading to a very large island size or ergodisation. Consequently, EC current drive must be applied to compensate the bootstrap current. For the X2-mode scenarios, the ECCD efficiency is fairly high: the maximum ECCD with $10 \mathrm{MW}$ is $142 \mathrm{kA}$ and $92 \mathrm{kA}$ for the "low-" and the "high-mirror" case, respectively, for optimum launch angles. Whereas the ECCD profile is very close to the $j_{b}$ profile for the "high-mirror" case allowing for a nearly local compensation with only small deviation from the currentless $t(r)$, the central counter-ECCD in the "low-mirror" case reduces significantly $t(r)$ at the inner radii. In principle, a proper bootstrap current control by ECCD can reduce the time to achieve stationary conditions to several skin-times [18].

\section{SUMMARY AND CONCLUSIONS}

Two different momentum correction techniques based on the three mono-energetic transport coefficients calculated by the DKES code have been described. Both correction techniques are very attractive and have been implemented in the energy convolution algorithm of the mono-energetic transport coefficients which are obtained by interpolation from precalculated databases for each magnetic configuration under investigation. This approach turns out to be very efficient and can be easily used for predictive transport modelling. 
The first of these correction techniques solves a generalised Spitzer problem for the parallel flows, i.e. for the bootstrap current as well as for the parallel electric conductivity. The impact of momentum conservation for the radial transport in the stellarator lmfp-regimes is shown (in Sec. II B) to be negligible and, consequently, has been disregarded. For axisymmetric configurations as well as for the higher collisionaltiy regimes in stellarators, however, corrections to the radial transport are not available using this technique.

The second correction technique is based on a moment equation expansion of the drift-kinetic equation (a traditional method e.g. for tokamaks). In this approach, the symmetric contributions with respect to $v_{\|}$, i.e. the radial transport, are linked to the parallel flows and momentum correction affects both contributions which is the main advantage of this approach compared to the first technique. Furthermore, this approach is numerically very fast since only a small system of linear equations (number of particle species times number of Sonine polynomials) has to be solved in the energy convolution algorithm. So far, this technique is implemented only for three Sonine polynomials; further benchmarking is needed, e.g. with the technique described in Refs. [13, 14].

The benchmarking of both momentum correction techniques showed very good agreement. In particular for tokamaks, where several models are available both for the parallel electric conductivity and for the bootstrap current density, very reasonable agreement for all approaches is obtained. Density gradients increase the corrected bootstrap current density whereas temperature gradients reduce it with respect to the uncorrected value. To take all the profile effects into consideration, the total bootstrap currents with and without momentum correction have been calculated for both the "low-" and "high-mirror" configuration of the W7-X stellarator. The predictive transport simulations (mainly with neoclassical transport modelling) showed a moderate reduction of the total bootstrap current roughly of the order of $20 \%$ of the uncorrected value. Consequently, the minimisation of the mono-energetic bootstrap current coefficient, which is an optimisation criterium of W7-X, is supported by an evaluation based on a momentum-conserving collision operator.

The parallel ion flow velocities found in the stellarator simulations are very small so that the assumption of an unshifted Maxwellian for the Oth-order distribution function is validated. The radial electric fields, however, can become fairly large both for electron-root scenarios and in the plasma periphery where maximum pressure gradients occur. For very large $E_{r}$, the traditional neoclassical ordering is violated for ions and impurities. First, the mono-energetic treatment, which is based on the approximation of an incompressible $\mathbf{E} \times \mathbf{B}$ drift, is not possible and, furthermore, density and potential variations within the flux surfaces must be included. Second, the radial transport of ion parallel momentum might become important, which is in conflict with the local approach. Consequently, the treatment of very large radial electric fields is far beyond the traditional neoclassical ordering in stellarators, which is mandatory for the concept of precalculated databases with local and mono-energetic transport coefficients as the basis for the momentum correction techniques. The situation is different in tokamaks, where a rigorous treatment is possible [41].

The "direct" momentum correction technique solving the generalised Spitzer problem is also used for the momentum correction for electron cyclotron current drive, ECCD [35] (but not implemented for the neutral beam current drive, NBCD, so far). Consequently, advanced tools for the simulation of current control scenarios by ECCD for the W7-X stellarator (which has no ohmic transformer) are now available. Since the long-pulse operation of W7-X relies on the island divertor concept, the control of the island configuration at the plasma edge and, consequently, the total plasma current is mandatory. The analysis of W7-X discharge scenarios up to quasi-stationary time scales is the natural next step for further investigations.

\section{Acknowledgments}

The authors acknowledge helpful discussions with N.B. Marushchenko and P. Helander, and in particular with C. Angioni and O. Sauter with respect to the benchmarking of the tokamak scenarios.

\section{APPENDIX A: LINEARISED COLLISION TERM WITH MOMENTUM CONSERVATION}

Here, the $1 s t$ Legendre term of the linearised collision operator, $C_{l=1}^{\alpha}$, is briefly documented. The basic formulation of the Fokker-Planck collision operator and the definitions of the Rosenbluth potential can be found e.g. in Refs. [42, 43]. Here, the integro-differential equation (18) is formulated with respect to the Spitzer function, $S^{\alpha}$, of the species $\alpha$, i.e. the Maxwellians are dropped $\left(K^{\alpha}(x)=S^{\alpha}(x) \exp \left(-x^{2}\right)\right)$. This procedure allows for higher accuracy in the numerical solution at large velocities.

$$
C_{l=1}^{\alpha}\left(K^{\alpha}\right)=\nu_{0}^{\alpha} e^{-x^{2}} \sum_{\beta} \frac{n^{\beta}\left(Z^{\beta}\right)^{2}}{n^{\alpha}\left(Z^{\alpha}\right)^{2}}\left(c_{2}^{\alpha \beta} \frac{d^{2}}{d x^{2}} S^{\alpha}+c_{1}^{\alpha \beta} \frac{d}{d x} S^{\alpha}+c_{0}^{\alpha \beta} S^{\alpha}+I^{\alpha \beta}\right)
$$

where $x \equiv x^{\alpha}=v / v_{t h}^{\alpha}\left(x^{\beta}=x v_{t h}^{\alpha} / v_{t h}^{\beta}\right), \nu_{0}^{\alpha}=n^{\alpha}\left(Z^{\alpha} e^{2} / \epsilon_{0} m^{\alpha}\right)^{2} \ln \Lambda / 4 \pi\left(v_{t h}^{\alpha}\right)^{3}$ is the thermal collision frequency, $Z^{\alpha}$ is the charge state, $\epsilon_{0}$ is the dielectric constant, and $\ln \Lambda$ is the Coulomb logarithm. The coefficients for the diffusive part are given 


$$
\begin{aligned}
& \begin{aligned}
c_{2}^{\alpha \beta} & =\left(\frac{v_{t h}^{\beta}}{v_{t h}^{\alpha}}\right)^{2} \frac{\eta\left(x^{\beta}\right)}{2 x^{2}} \\
c_{1}^{\alpha \beta} & =\frac{1}{x^{2}}\left(\frac{m^{\alpha}}{m^{\beta}}\left(1-\frac{T^{\beta}}{T^{\alpha}}\left(2+\frac{1}{2 x^{2}}\right)\right) \eta\left(x^{\beta}\right)+\frac{2 x^{\beta}}{\sqrt{\pi}} e^{-\left(x^{\beta}\right)^{2}}\right) \\
c_{0}^{\alpha \beta} & =-\frac{\widehat{\eta}\left(x^{\beta}\right)}{x^{3}}-2 \frac{m^{\alpha}}{m^{\beta}}\left(1-\frac{T^{\beta}}{T^{\alpha}}\right)\left(\frac{\eta\left(x^{\beta}\right)}{x}-\frac{2}{\sqrt{\pi}}\left(\frac{v_{t h}^{\alpha}}{v_{t h}^{\beta}}\right)^{3} e^{-\left(x^{\beta}\right)^{2}}\right) \\
\text { with } \quad \eta(x) & =\operatorname{erf}(x)-\frac{2 x}{\sqrt{\pi}} e^{-x^{2}} \quad \text { and } \quad \widehat{\eta}(x)=\operatorname{erf}(x)-\frac{\eta(x)}{2 x^{2}},
\end{aligned}
\end{aligned}
$$

and for the integral contributions (from the $1 s t$ Legendre coefficients of the Rosenbluth potentials)

$$
\begin{aligned}
x^{2} \cdot I^{\alpha \beta}=\frac{4}{\sqrt{\pi}} \frac{m^{\alpha}}{m^{\beta}} \frac{v_{t h}^{\alpha}}{v_{t h}^{\beta}}\left(x^{\beta}\right)^{2} K^{\beta}\left(x^{\beta}\right)-\frac{8}{3 \sqrt{\pi}}\left(2 \frac{m^{\alpha}}{m^{\beta}}-1\right) \frac{v_{t h}^{\beta}}{v_{t h}^{\alpha}} \int_{0}^{x^{\beta}}\left(x^{\prime \beta}\right)^{3} K^{\beta}\left(x^{\prime \beta}\right) d x^{\prime \beta} \\
\quad+\frac{16}{5 \sqrt{\pi}}\left(\frac{v_{t h}^{\beta}}{v_{t h}^{\alpha}}\right)^{3} \int_{0}^{x^{\beta}}\left(x^{\prime \beta}\right)^{5} K^{\beta}\left(x^{\prime \beta}\right) d x^{\prime \beta}+\frac{8}{3 \sqrt{\pi}}\left(\frac{m^{\alpha}}{m^{\beta}}-2+\frac{6}{5} x^{2}\right) x\left(x^{\beta}\right)^{2} \int_{x^{\beta}}^{\infty} K^{\beta}\left(x^{\prime \beta}\right) d x^{\prime \beta}
\end{aligned}
$$

where $K^{\beta}=S^{\beta} \exp \left(-\left(x^{\beta}\right)^{2}\right)$. All these terms in eqs. (A1) to (A3) are needed to guarantee the like-particle momentum conservation (with only $\beta=\alpha$ contributions included)

$$
\int_{0}^{\infty} x^{3} C_{l=1}^{\alpha}\left(K^{\alpha}\right) d x=0
$$

Now, the cross-coupling terms $\beta \neq \alpha$ are briefly analysed. For the electron-ion terms $(\alpha=e$ and $\beta=i$ ), the coefficients in leading order of the mass ratio are

$$
c_{2}^{e i} \sim c_{1}^{e i}=\mathcal{O}\left(\frac{m^{e}}{m^{i}}\right) \simeq 0 \quad \text { and } \quad c_{0}^{e i}=-\frac{1}{x^{3}}
$$

which leads (with the summation) to $c_{0}^{e} \simeq-\left(\widehat{\eta}(x)+Z_{\mathrm{eff}}\right) / x^{3}$, the (normalised) pitch-angle collision frequency. The leading term of the integral contributions is

$$
x^{2} \cdot I^{e i} \approx \frac{8}{3 \sqrt{\pi}} \frac{v_{t h}^{i}}{v_{t h}^{e}} \int_{0}^{\infty}\left(x^{i}\right)^{3} K^{i} d x^{i} \simeq \frac{4}{3} \frac{\left\langle V_{\|}^{i}\right\rangle}{v_{t h}^{e}} .
$$

Note that the additional $v$ for the estimation of the parallel flows ( $\int v_{\|} \mathcal{F} v^{2} d v$ ) leads to the relation (in normalised form) $v_{t h}^{i} K^{i} \sim v_{t h}^{e} K^{e}$, i.e. $I^{e i} \sim \mathcal{O}(1)$. Consequently, the integral part $I^{e i}$ forces the electrons to follow the parallel ion flow, $V_{\|}^{i}$. The ion-electron coupling terms in leading order are given by

$$
c_{2}^{i e} \sim c_{1}^{i e} \sim c_{0}^{i e}=\mathcal{O}\left(\sqrt{\frac{m^{e}}{m^{i}}}\right) \simeq 0
$$

and for the integral contribution

$$
I^{i e} \approx \frac{8}{3 \sqrt{\pi}} \frac{T^{i}}{T^{e}} x \int_{0}^{\infty} K^{e} d x^{e}=\mathcal{O}\left(\sqrt{\frac{m^{e}}{m^{i}}}\right) \simeq 0
$$

i.e. the coupling of the ions to the electrons is rather weak. All like-particle terms $I^{\alpha \alpha}$ are taken into account, whereas for the integral contributions $I^{e i}$ and $I^{i e}$ only the leading order terms are included. Furthermore, impurity species are only included in the diffusive terms, but omitted for the integral contributions.

The diffusive contributions in the sytem of eq. (18) are simply integrated by a difference scheme with the integral contributions $I^{\alpha \beta}$ estimated within direct iteration. The convergence is rather fast: typically about 10 iteration steps are quite sufficient. It is important that the difference scheme for calculating $C_{l=1}^{\alpha}$ on the r.h.s. of eq. (18) is identical to the integration scheme for the 1.h.s. to guarantee sufficient accuracy. 


\section{APPENDIX B: AXISYMMETRIC TOKAMAK - INTRINSIC AMBIPOLARITY}

For the axisymmetric tokamak, the mirror term in the Vlasov operator in eq. (5) is related to the radial component of the $\nabla B$-drift, $\dot{r}=\mathbf{v}_{\nabla B} \cdot \nabla r$; see eq. (7). Neglecting the $\mathbf{E} \times \mathbf{B}$ drift in the Vlasov operator, the relation

$$
\mathcal{V}\left(\frac{p}{B} A\right)=\frac{p}{B} \mathcal{V}(A)-A \frac{v\left(1+p^{2}\right)}{2 B^{3}} \mathbf{B} \cdot \nabla B
$$

holds for arbitrary $A$. Using this relation in eqs. (2) with the definition of $\dot{r}$, taking the $p$ moment and flux-surface-averaging leads to a link between the mono-energetic transport coefficients

$$
\begin{aligned}
& D_{11}^{\alpha}=\frac{1}{t \epsilon_{t}} \frac{m^{\alpha}}{q^{\alpha} B_{0}}\left\{\frac{1}{\left\langle b^{2}\right\rangle} \nu^{\alpha} D_{31}^{\alpha}+\frac{2}{3} v_{d} R B_{0} \nu^{\alpha}\left\langle\frac{\widetilde{G}}{B}\right\rangle\right\} \\
& D_{13}^{\alpha}=\frac{1}{t \epsilon_{t}} \frac{m^{\alpha}}{q^{\alpha} B_{0}}\left\{\frac{1}{\left\langle b^{2}\right\rangle} \nu^{\alpha} D_{33}^{\alpha}-\frac{v^{2}}{3}\right\}
\end{aligned}
$$

where $v_{d}=m^{\alpha} v^{2} / 2 q^{\alpha} R B_{0}$ is the characteristic drift velocity and $R$ the major radius. With the Onsager symmetry relation, $D_{13}^{\alpha}=-D_{31}^{\alpha}$, only one independent transport coefficient exists.

The solution for the parallel flow moments defined in eq. (20) can be shown to have the form

$$
n^{\alpha}\left\langle b U_{j}^{\alpha}\right\rangle=X_{j}^{\alpha}+\frac{n^{\alpha} E_{r}}{t \epsilon_{t} B_{0}} \delta_{0 j}
$$

where the $X_{j}^{\alpha}$ are independent of $E_{r}$, i.e. the radial electric field affects only the parallel particle flow. With this result, also the radial particle flux becomes independent of $E_{r}$

$$
\begin{aligned}
& \frac{\Gamma^{\alpha}}{n^{\alpha}}=-\frac{\langle\mathbf{E} \cdot \mathbf{B}\rangle}{t \epsilon_{t}\left\langle B^{2}\right\rangle}-\frac{1}{t \epsilon_{t}} \frac{m^{\alpha}}{q^{\alpha} B_{0}} \\
& \frac{1}{\left\langle b^{2}\right\rangle} \sum_{\beta \neq \alpha} \sum_{j=0}^{j_{x}} c_{j}\left(\frac{X_{j}^{\alpha}}{n^{\alpha}} \frac{M_{0 j}^{\alpha \beta}}{\tau^{\alpha \beta}}+\frac{X_{j}^{\beta}}{n^{\beta}} \frac{N_{0 j}^{\alpha \beta}}{\tau^{\alpha \beta}}\right) \\
& +\frac{m^{\alpha}}{q^{\alpha}} \frac{\left\langle\widetilde{G}^{2}\right\rangle}{B_{0}^{2}}\left\{\sum_{\beta \neq \alpha}\left(\frac{\left(n^{\alpha} T^{\alpha}\right)^{\prime}}{n^{\alpha} q^{\alpha}}-\frac{\left(n^{\beta} T^{\beta}\right)^{\prime}}{n^{\beta} q^{\beta}}\right) \frac{M_{00}^{\alpha \beta}}{\tau^{\alpha \beta}}-\sum_{\beta \neq \alpha}\left(\frac{\left(T^{\alpha}\right)^{\prime}}{q^{\alpha}} \frac{M_{01}^{\alpha \beta}}{\tau^{\alpha \beta}}+\frac{\left(T^{\beta}\right)^{\prime}}{q^{\beta}} \frac{N_{01}^{\alpha \beta}}{\tau^{\alpha \beta}}\right)\right\}
\end{aligned}
$$

where $\langle\mathbf{E} \cdot \mathbf{B}\rangle$ represents the parallel force driving the Ware pinch flux. Using the following identities for the matrix elements of the friction coefficients defined in eq. (21)

$$
\frac{M_{00}^{\alpha \beta}}{\tau^{\alpha \beta}}=\frac{n^{\beta} m^{\beta}}{n^{\alpha} m^{\alpha}} \frac{M_{00}^{\beta \alpha}}{\tau^{\beta \alpha}} \quad \text { and } \quad \frac{M_{0 j}^{\alpha \beta}}{\tau^{\alpha \beta}}=-\frac{n^{\beta} m^{\beta}}{n^{\alpha} m^{\alpha}} \frac{N_{0 j}^{\beta \alpha}}{\tau^{\beta \alpha}}
$$

straightforward calculation leads to intrinsic fulfilment of the ambipolarity constraint of the radial particle fluxes, $\sum_{a} q^{\alpha} \Gamma^{\alpha}=$ 0 .

[1] S.P. Hirshman, K.C. Shaing, W.I. van Rij, C.O. Beasley, Jr., and E.C. Crume, Phys. Fluids 29, 2951 (1986).

[2] W.I. van Rij and S.P. Hirshman, Phys. Fluids B 1, 563 (1989).

[3] V. Tribaldos, Phys. Plasmas 8, 1229 (2001).

[4] A. Wakasa, S. Murakami, H. Maßßberg, C. D. Beidler, N. Nakajima, K. Watanabe, H. Yamada, M. Okamoto, S. Oikawa, and M. Itagaki, J. Plasma Fusion Res. SERIES 4, 408 (2001).

[5] S. Murakami, A. Wakasa, H. Maßßberg, C.D. Beidler, H. Yamada, and LHD experimental group, Nucl. Fusion 42, L19 (2002).

[6] M.Yu. Isaev, S. Brunner, W.A. Cooper, T.M. Tran, A. Bergmann, C.D. Beidler, J. Geiger, H. Maaßberg, J. Nührenberg, and M. Schmidt, Fusion Sci. Techn. 50, 440 (2006).

[7] K. Allmaier, S.V. Kasilov, W. Kernbichler, and G.O. Leitold, Phys. Plasmas 15, 072512 (2008).

[8] C.D. Beidler, M.Yu. Isaev, S.V. Kasilov, W. Kernbichler, H. Maaßberg, D. S. Murakami, V.V. Nemov, M. Schmidt, D.A. Spong, V. Tribaldos, and A. Wakasa, Proc. 22nd IAEA Fusion Energy Conf., Geneve, 2008; www-pub.iaea.org/MTCD/Meetings/fec2008pp.asp th_p8-10.pdf.

[9] S.P. Hirshman, Phys. Fluids 21, 224 (1978).

[10] S.P. Hirshman and D.J. Sigmar, Nucl. Fusion 21, 1079 (1981). 
[11] K.C. Shaing and J.D. Callen, Phys. Fluids 26, 3315 (1983).

[12] M. Taguchi, Phys. Fluids B 4, 3638 (1992).

[13] H. Sugama and S. Nishimura, Phys. Plasmas 9, 4637 (2002).

[14] H. Sugama and S. Nishimura, Phys. Plasmas 15, 042502 (2008).

[15] L. Spitzer and R. Härm, Phys. Rev. 89, 977 (1953).

[16] M. Taguchi, Plasma Phys. Control. Fusion 30, 1897 (1988).

[17] M. Taguchi, Plasma Phys. Control. Fusion 31, 241 (1989).

[18] Yu. Turkin, H. Maßßberg, C.D. Beidler, J. Geiger, and N.B. Marushchenko, Fusion Sci. Techn. 50, 387 (2006).

[19] C.D. Beidler, M.Yu. Isaev, S.V. Kasilov, W. Kernbichler, H. Maßßberg, S. Murakami, V.V. Nemov, D.A. Spong, and V. Tribaldos, Proc. 17th Int. Toki Conf. and 16th Int. Stellarator/Heliotron Workshop, Toki, 2007, edited by T. Morisaki, www.nifs.ac.jp/itc/itc17, P2-031.

[20] S.P. Hirshman, W.I. van Rij, and P. Merkel, Comp. Phys. Comm. 43, 143 (1986).

[21] J. Nührenberg and R. Zille, Theory of Fusion Plasmas (Varenna 1987), Editrice Compositori, Bologna (1988), p. 3.

[22] F.L. Hinton and R.D. Hazeltine, Rev. Mod. Physics 48, 239 (1976).

[23] P. Helander and A.N. Simakov, Phys. Rev. Lett. 101, 145003 (2008).

[24] F.L. Hinton and M.N. Rosenbluth, Phys. Fluids 16, 836 (1973).

[25] A.A. Galeev and R.Z. Sagdeev, Review of Plasma Physics 7, 257 (1979).

[26] C.D. Beidler and W.D. D'haeseleer, Plasma Phys. Control. Fusions 37, 463 (1995).

[27] M. Romé, V. Erckmann, U. Gasparino, and N. Karulin, Plasma Phys. Control. Fusion 40, 511 (1998).

[28] S.P. Hirshman, Phys. Fluids 23, 1238 (1980).

[29] O. Sauter, C.Angioni, and Y.R. Lin-Liu, Phys. Plasmas 6, 2834 (1999).

[30] O. Sauter, R.J. Buttery, R. Felton, T.C. Hender, D.F. Howell, and contributors to the EFDA-JET Workprogramme, Plasma Phys. Contr. Fusion 44, 1999 (2002).

[31] S.P. Hirshman, Phys. Fluids 31, 3150 (1988).

[32] H. Maaßberg, R. Burhenn, U. Gasparino, G. Kühner, H. Ringler, and K.S. Dyabilin, Phys. Fluids B 5, 3627 (1993).

[33] H. Maßßberg, C.D. Beidler, U. Gasparino, M. Romé, the W7-AS Team, K.S. Dyabilin, N.B. Marushchenko, and S. Murakami, Phys. Plasmas 7, 295 (2000).

[34] A. Dinklage, H. Maaßberg, R. Preuss, Yu.A. Turkin, H. Yamada, E. Ascasibar, C.D. Beidler, H. Funaba, J.H. Harris, A. Kus, S. Murakami, S. Okamura, F. Sano, U. Stroth, Y. Suzuki, J. Talmadge, V. Tribaldos, K.Y. Watanabe, A. Werner, A. Weller, and M. Yokoyama, Nucl. Fusion 47, 1265 (2007).

[35] N.B. Marushchenko, C.D. Beidler, and H. Maßßberg, Fusion Sci. Techn. 55, 180 (2009).

[36] N.B. Marushchenko, H. Maaßberg, C.D. Beidler, V. Erckmann, J. Geiger, H.P. Laqua, A.G. Shalashov, E.V. Suvorov, Yu. Turkin, and W7-X Team, ECRH scenarios on W7-X, Proc. 7th Intern. Workshop “Strong Microwaves: Sources and Applications”, Nizhny Novgorod, 2008, edited by A.G. Litvak (Institute of Applied Physics, Nizhny Novgorod, 2009, in press).

[37] M. Yokoyama, H. Maßßberg, C.D. Beidler, V. Tribaldos, K. Ida, F. Castejón, T. Estrada, A. Fujisawa, T. Minami, T. Shimozuma, Y. Takeiri, J. Herranz, S. Murakami, and H. Yamada, Fusion Sci. Techn. 50, 327 (2006).

[38] P.I. Strand and W.A. Houlberg, Phys. Plasmas 8, 2782 (2001).

[39] D. Sharma, Y. Feng, F. Sardei, and D. Reiter, Nucl. Fusion 45, 825 (2005).

[40] D. Sharma, Y. Feng, and F. Sardei, Nucl. Fusion 46, S127 (2006).

[41] F.L. Hinton and S.K. Wong, Phys. Fluids 28, 3082 (1985).

[42] J. Killeen, A.A. Mirin, and M.E. Rensink, in Methods in Computational Physics, edited by J. Killeen (Academic Press, New York), 1976, Vol. 16.

[43] C.F.F. Karney, Comp. Phys. Rep. 4, 183 (1986). 